Draft VERSion March 2, 2018

Preprint typeset using LATEX style AASTeX6 v. 1.0

\title{
PROPERTIES OF A SMALL-SCALE SHORT-DURATION SOLAR ERUPTION WITH A DRIVEN SHOCK
}

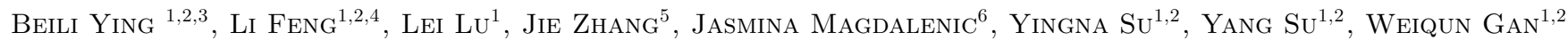 \\ ${ }^{1}$ Key Laboratory of Dark Matter and Space Astronomy, Purple Mountain Observatory, Chinese Academy of Sciences,210008 Nanjing, China \\ ${ }^{2}$ School of Astronomy and Space Science, University of Science and Technology of China, Hefei, Anhui 230026, China \\ ${ }^{3}$ Max-Planck-Institut für Sonnensystemforschung, Justus-von-Liebig-Weg 3, D-37077, Göttingen, Germany \\ ${ }^{4}$ State Key Laboratory of Space Weather, National Space Science Center, Chinese Academy of Sciences, Beijing 100190, China \\ ${ }^{5}$ Department of Physics and Astronomy, George Mason University, Fairfax, VA 22030, USA \\ ${ }^{6}$ Royal Observatory of Belgium, Belgium
}

\begin{abstract}
Large-scale solar eruptions have been extensively explored over many years. However, the properties of small-scale events with associated shocks have been rarely investigated. We present the analyses of a small-scale short-duration event originating from a small region. The impulsive phase of the M1.9-class flare lasted only for four minutes. The kinematic evolution of the CME hot channel reveals some exceptional characteristics including a very short duration of the main acceleration phase $(<2$ minutes), a rather high maximal acceleration rate $\left(\sim 50 \mathrm{~km} \mathrm{~s}^{-2}\right)$ and peak velocity $\left(\sim 1800 \mathrm{~km} \mathrm{~s}^{-1}\right)$. The fast and impulsive kinematics subsequently results in a piston-driven shock related to a metric type II radio burst with a high starting frequency of $\sim 320 \mathrm{MHz}$ of the fundamental band. The type II source is formed at a low height of below $1.1 \mathrm{R}_{\odot}$ less than $\sim 2$ minutes after the onset of the main acceleration phase. Through the band split of the type II burst, the shock compression ratio decreases from 2.2 to 1.3, and the magnetic field strength of the shock upstream region decreases from 13 to 0.5 Gauss at heights of 1.1 to $2.3 \mathrm{R}_{\odot}$. We find that the CME $\left(\sim 4 \times 10^{30} \mathrm{erg}\right)$ and flare $\left(\sim 1.6 \times 10^{30} \mathrm{erg}\right)$ consume similar amount of magnetic energy. The same conclusion for large-scale eruptions implies that small- and large-scale events possibly share the similar relationship between CMEs and flares. The kinematic particularities of this event are possibly related to the small footpoint-separation distance of the associated magnetic flux rope, as predicted by the Erupting Flux Rope model.
\end{abstract}

Keywords: Sun: corona - Sun: coronal mass ejections (CMEs) - Sun: flare - Sun: flux rope - Sun: shock wave

\section{INTRODUCTION}

Coronal mass ejections (CMEs) and flares are two most important eruptive phenomena in the atmosphere of the Sun, and are now regarded as the primary solar drivers of geomagnetic storms and ionospheric disturbances on the Earth. Theoretically and observationally, many researches found that a CME may originate as a magnetic flux rope (MFR) which contains a coherent magnetic structure with magnetic field winding around its central axis (e.g., Chen 1989; Zhang et al. 2012; Wang et al. 2015). In the Atmospheric Imaging Assembly (AIA; Lemen et al. 2012) field of view (FOV), MFRs can often be observed in $131 \AA$ and/or $94 \AA$ passbands (e.g., Cheng et al. 2013, 2014). Using AIA multi-wavelength observations of a flaring active region, Reeves \& Golub (2011) has constrained the temperature of MFRs to be between $5 \mathrm{MK}$ and $20 \mathrm{MK}$, which suggests that the MFRs could

lfeng@pmo.ac.cn reach flare-like temperatures. When MFRs are observed edge-on above the limb, they show up as dark cavities characterized by low densities and high temperatures (Gibson \& Fan 2006; Gibson et al. 2010; Kucera et al. 2012), exhibiting signatures of spiral motion of plasma (see details in Schmit et al. 2009). Due to the property of its high temperature, the MFR observed in the AIA images is also termed as a "hot channel (HC)" (Zhang et al. 2012). When the HC transits into the FOV of the Large Angle Spectroscopic Coronagraph (LASCO; Brueckner et al. 1995), it often appears as a three-part CME structure (Illing \& Hundhausen 1983).

An MFR can form either before or during an eruption. Many researches have found indirect evidences of the existence of MFR before the eruption, e.g., forward or reverse S-shaped sigmoids (Rust \& Kumar 1996; Canfield et al. 1999; Tripathi et al. 2009), dark cavities (Low \& Hundhausen 1995; Gibson et al. 2004; Dove et al. 2011), as well as filaments (Mackay et al. 2010; Guo et al. 2010; 
Su et al. 2011). Patsourakos et al. (2013) presented the first direct evidence of a fast CME driven by the destabilization of a preformed coronal MFR. Song et al. (2014) witnessed the formation of an MFR during the eruption. Theoretically, CMEs have been presumed as the eruption of MFRs: some began with initial coronal FRs in equilibrium (Chen 1989; Vršnak 1990; Chen \& Garren 1993; Gibson \& Low 1998; Roussev et al. 2003), while others started with the emergence of FRs from below the photosphere (Wu et al. 1997; Fan \& Gibson 2003; Manchester et al. 2004). Among the existing CME models, the semi-analytic erupting flux-rope (EFR) model of CMEs (Chen 1989; Chen \& Kunkel 2010) has been tested widely. The basic physical principle of the EFR model is that the major radial Lorentz self-force (the Lorentz "hoop force") accelerates coronal FRs. The EFR model introduced the distance $S_{f}$ between the two footpoints of the MFR at the base of the corona. It is suggested that $S_{f}$ is a key parameter to determine the dynamics of MFRs.

When the speed of a CME is higher than the local fast magnetosonic speed, a shock wave may be driven. If the CME as a driver pushes the plasma and its body expands in all directions; meanwhile, the offset distance between shock front and CME ejecta always increases in time, this type of shock can be regarded as a threedimensional piston-driven shock (Vršnak \& Cliver 2008). Shock waves are accompanied by a type II radio burst observed in the radio dynamic spectra as emission lanes slowly drifting to lower frequencies with time. The type II bursts classically consist of two bands separated by approximately a factor of 2 in frequency. The lower frequency (fundamental) band is approximately at the local plasma frequency $f_{p}$, while the upper (second harmonic) band is roughly at $2 f_{p}$. The starting frequency of the metric type II emission associated with the coronal shock is usually less than $300 \mathrm{MHz}$ with an average of about $100 \mathrm{MHz}$ (Gopalswamy et al. 2005). High starting frequency implies that the shock is propagating through a high density region and hence a low formation height. Some of the coronal shock waves propagate in the interplanetary space and become the source of type II radio radiation in the decametric to kilometric wave range (Gopalswamy et al. 2005; Lu et al. 2017; Prakash et al. 2017). In some of the type II burst events, fundamental and/or harmonic bands are split into two morphologically similar lanes (Nelson \& Melrose 1985). A number of explanations were suggested accounting for the magnetic, Doppler, and geometrical effects (Krüger 1979). Smerd et al. (1974) attributed the band split to the emission from the upstream and downstream shock regions, demonstrating a potentially vital method that could offer an estimation of the corona Alfvén velocity and magnetic field strength.
Mini-filaments are small-scale features in the solar atmosphere, which frequently occur across the entire disk. They have a spatial scale of a few tens of arcsecs and are the small-scale analog to large-scale filaments (Wang et al. 2000). Mini-filaments located at the edge of active regions often act as triggers of coronal jets (Hong et al. 2016; Wyper et al. 2017). It is rare that these mini-filaments are observed to be associated with CME HCs. In this paper we present the analyses of such a CME event and its driven shock. Recently, Kumari et al. (2017) analyzed the same CME-driven shock, and used the shock to represent the near-Sun kinematics of the CME. In our study, we have not only investigated the shock properties much more extensively, but also give the interpretations on what causes the particularities of this eruption event. We have quantified the characteristics of various eruption features: $\mathrm{CME} \mathrm{HC}$, its driven bright front as the likely location of the shock, and the associated flare. As the distance of footpoint separation of an MFR is suggested to be a key parameter to determine its dynamics, we will investigate how the analyzed eruption originating from a small source region differs from large-scale eruptions. Previous studies have found equal partition of free magnetic energies between flares and CMEs in large-scale eruption events (Emslie et al. 2012; Feng et al. 2013). Similar energy estimate will also be carried out to check whether our small-scale eruption follows the same rule.

The organization of the paper is as follows. In Section 2, we describe the observations of our small-scale eruptive events taken by different instruments. In Section 3 , the properties of the $\mathrm{HC}$ and associated bright front/shock front, the impulsive compact flare, the CME travelling into LASCO FOV are analyzed. The deduced coronal magnetic field is included as well. The last section summarizes the results and discusses the differences and similarities between small-scale and large-scale eruptions.

\section{INSTRUMENTS AND OBSERVATIONS}

\subsection{Instruments}

The Atmospheric Imaging Assembly (AIA) launched as a part of NASA's Solar Dynamics Observatory (SDO) mission provides high temporal (12 seconds) and spatial resolution (0.6" per pixel) full-disk images of the corona at multiple wavelengths covering the temperature from $\sim 5000 \mathrm{~K}$ to $\sim 20 \mathrm{MK}$. The vector magnetogram data from the Helioseismic and Magnetic Imager (HMI) (Scherrer et al. 2012) aboard the SDO reveals the magnetic field context of the source region of the eruption event. To examine the Soft X-ray (SXR) emission of the associated flare, we study the data from $\mathrm{Geo}$ stationary Operational Environmental Satellite (GOES), 


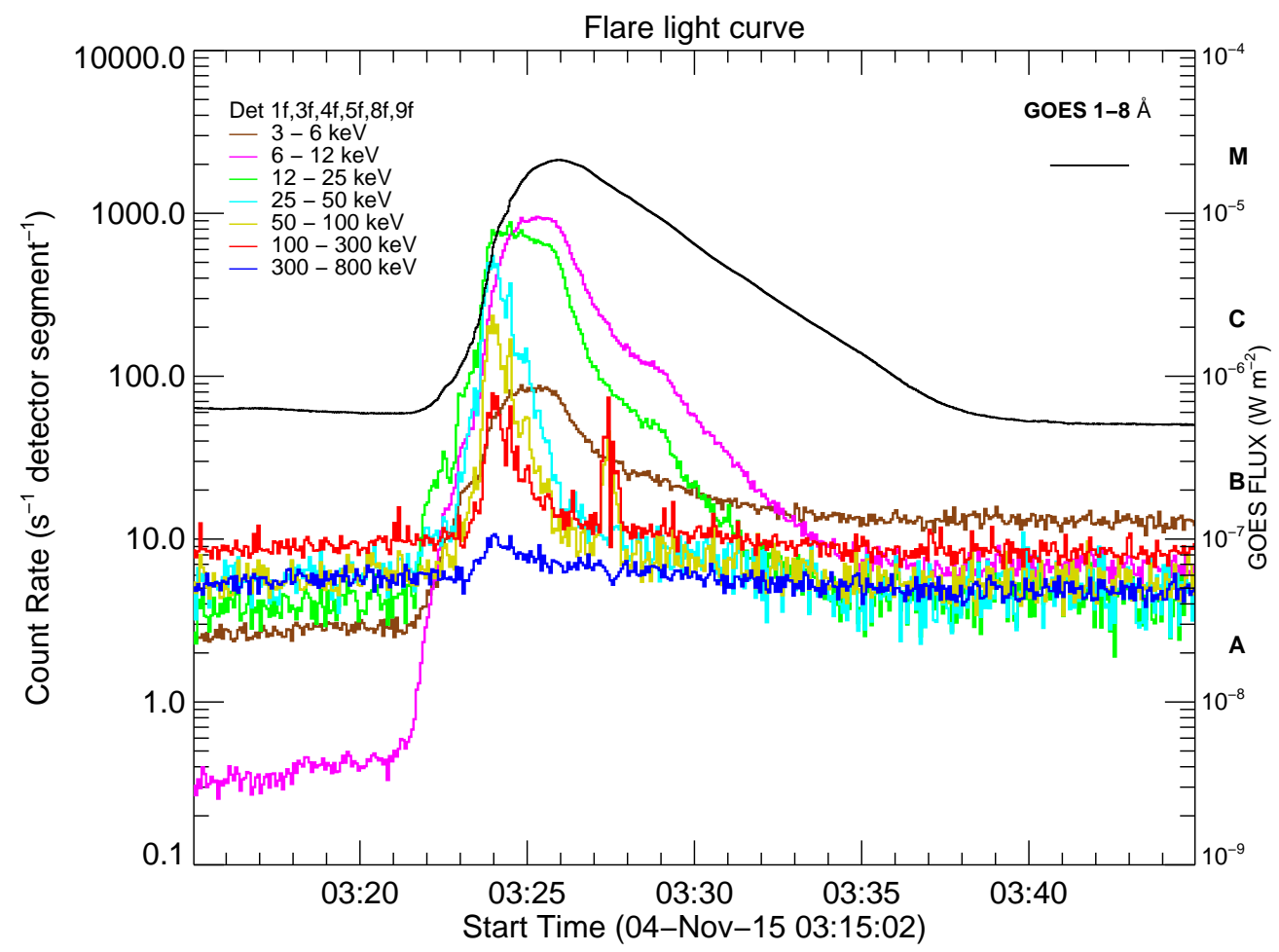

Figure 1: The light curves of GOES 1-8 $\AA$ and RHESSI in multiple energy bands up to $800 \mathrm{keV}$.

and discuss the Hard X-ray (HXR) emission through the Reuven Ramaty High-Energy Solar Spectroscopic Imager (RHESSI) mission (Lin et al. 2002). For the associated CME, we use the data from the Large Angle Spectroscopic Coronagraph (LASCO) C2 aboard the Solar and Heliospheric Observatory(SOHO) with the field of view (FOV) 1.5-6 $R_{\odot}$. In order to investigate the type II radio burst associated with the CME-driven shock, we utilize the radio dynamic spectra from Learmonth Observatory in Australia, with the frequency range of 25-180 $\mathrm{MHz}$, and the temporal resolution of 3 seconds, and from Culgoora observatory whose frequency range of 18-1800 $\mathrm{MHz}$.

\subsection{Event Overview}

On November 4, 2015, an eruption occurred from a small region at the edge of NOAA active region 12445 $\left(\mathrm{W} 70^{\circ}, \mathrm{N} 15^{\circ}\right)$ located close to the west solar limb. The GOES 1-8 A SXR flux indicated by the black line in Figure 1 shows that the M1.9-class flare in this event has a short duration of about 18 minutes, and its flux starts to rise at 03:21 UT and peaks at 03:26 UT. RHESSI photon light curves in different energy bands within 3$800 \mathrm{keV}$ are also presented in Figure 1. The enhancement of the photon count rates in the high energy band of $300-800 \mathrm{keV}$ implies that the electrons were probably accelerated to high energies.

Figure 2 is an overview of the AIA observations of the event including the evolution of the mini-filaments (a-c),
CME HC (d-i), and a bright front preceding the HC (fi). Panels (a-c) present the evolution of mini-filaments in AIA $211 \AA(\sim 2 \mathrm{MK})$ and $304 \AA(\sim 0.05 \mathrm{MK})$ before the eruption at $\sim 02: 30 \mathrm{UT}$ and in $304 \AA \sim 03: 23 \mathrm{UT}$ during the eruption. The white box in panel (c) defines the region of the interest (ROI) enlarged in panels (a) and (b). Two white arrows mark the positions of two mini-filaments or their segments. Actually quite a few mini-filaments were observed in this event. As an example, in panel (a) one of the mini-filaments is delineated by a yellow dotted line. In panel (b), blue and yellow contours indicate the positive and negative radial magnetic fields of AR 12445 observed by HMI at 02:34:12 UT. As it is rather close to the limb, to reduce projection effects, the radial component derived from the HMI vector magnetogram instead of the line-of-sight magnetic field is plotted. As revealed by panel (c), the mini-filaments in the white box begins to evolve slowly at $\sim 03: 23 \mathrm{UT}$. We conjecture that these mini-filaments may be related to the eruption process.

Figure 2 (d)-(e) are AIA base-difference images in 94 $\AA(\sim 7 \mathrm{MK})$ at 03:23:00 UT and 03:24:00 UT produced by subtracting a pre-event image at 03:20:00 UT. In Figure 2 (d), the colors of HMI radial magnetic field contours have the same definition as in Figure 2 (b). The location of the flaring region is consistent with that of the RHESSI HXR source in 25-50 keV, marked by the red contours with levels of $30 \%, 40 \%, 50 \%, 70 \%, 90 \%$ of the 

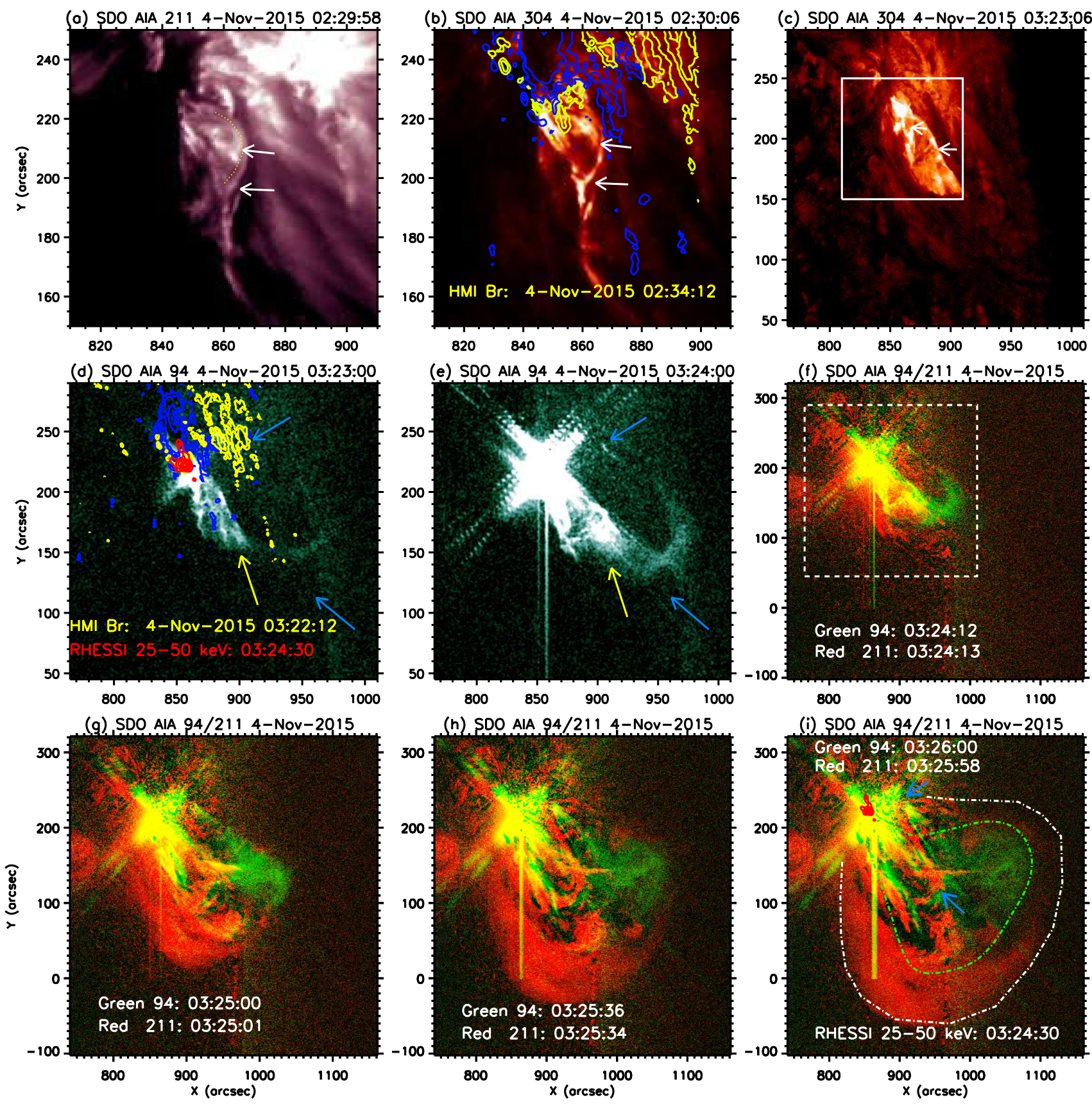

Figure 2: (a)-(c) Evolution of the mini-filaments as observed in AIA $211 \AA(\sim 2 \mathrm{MK})$ and $304 \AA(\sim 0.05 \mathrm{MK})$. The two white arrows mark the positions of two mini-filaments or their segments. The white box in panel (c) defines the region of the interest (ROI) enlarged in panels (a) and (b). Panel (c) is an AIA $304 \AA$ image observed at 03:23 UT at the beginning of the eruption, while panels (a) and (b) are AIA images observed in $304 \AA$ and $211 \AA$, respectively, at 02:30 UT before the eruption. In panel (a), one of the mini-filaments is delineated by a yellow dotted line. In panel (b), the blue (positive) and yellow contours (negative) show HMI radial magnetic field at 02:34 UT. (d)-(e) AIA $94 \AA$ ( 7 MK) base difference images displaying the eruption of the HC at 03:23:00 UT and 03:24:00 UT. Two blue arrows point to the same coordinates in panels (d), (e), (i). The upper arrow indicates a fixed footpoint of a coronal loop heated by the accelerated particles. The lower arrow shows the top evolution of the loop. The white box in panel (f) defines the ROI enlarged in panel (c), (d) and (e). SDO/HMI radial magnetic field (blue: positive and yellow: negative) is at 03:22:12 UT in panel (d). The contour levels of the RHESSI 25-50 keV source (red contours) are 30\%, 40\%, 50\%, 70\%, $90 \%$ in panels (d) and (i). The RHESSI source is at 03:24:30 UT around flare peak time. Yellow arrows represent the HC front in panels (d) and (e). (f)-(i) The synthesized base difference images from 03:24 UT to 03:26 UT with the $211 \AA$ image in the red channel and the $94 \AA$ image in the green channel. In panel (i), a green dotted-dashed profile indicates the periphery of the HC at 03:26:00 UT, and a white curve shows the periphery of the EUV bright front at 03:25:58 UT. For all the base difference images, a pre-event image at 03:20:00 UT is subtracted. 

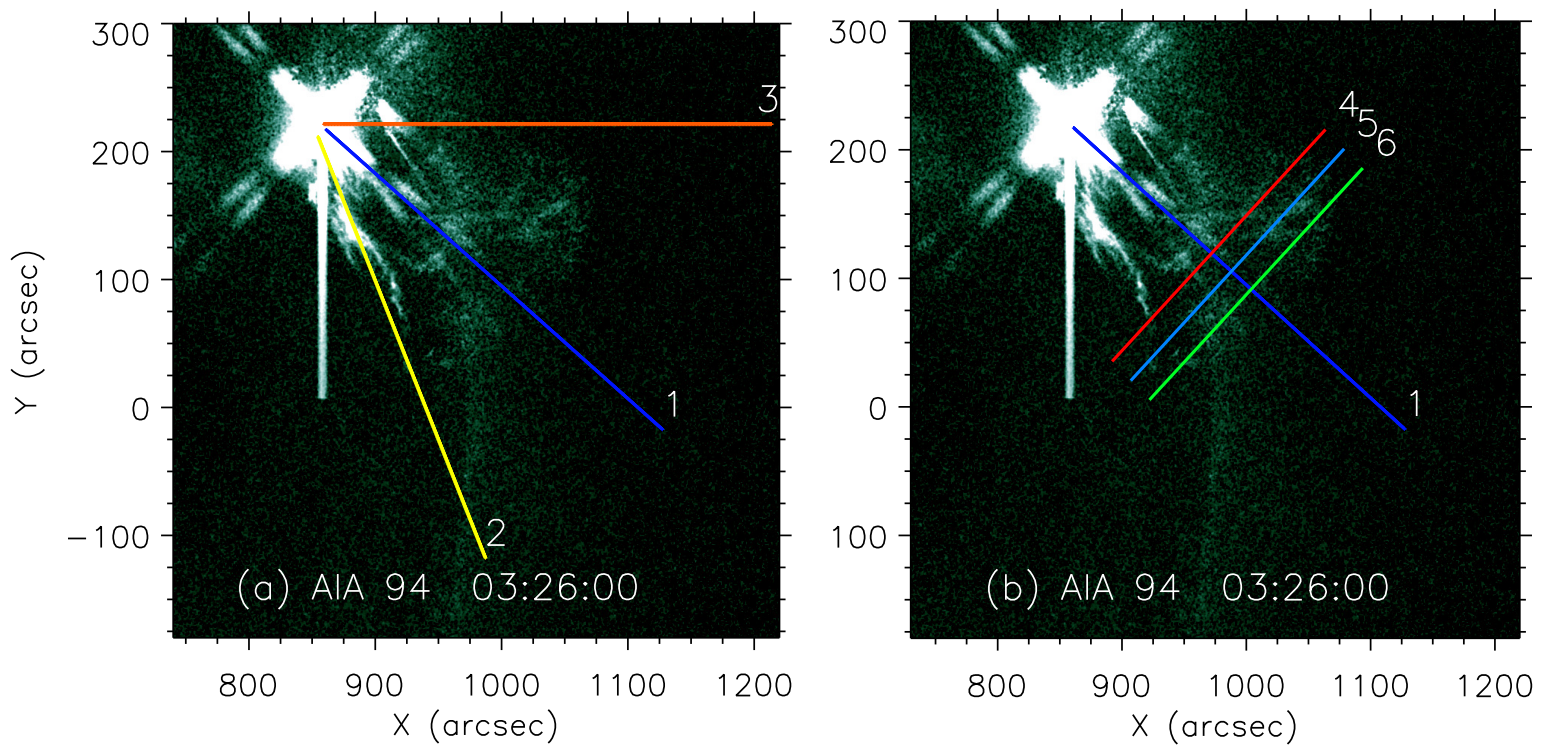

Figure 3: SDO/AIA $94 \AA$ ( $\sim 7 \mathrm{MK})$ base difference images displaying the eruption of the HC at 03:26:00 UT. In panel (a), solid lines 1, 2 mark two selected slices along the propagation direction of the HC nose and its flank. Slice 3 is chosen to track the $\mathrm{HC}$ deflection. The other lines 4-6 represent three slices along the expansion directions of the HC flank in panel (b). The width of slice 3 is 7 arcseconds and that of other slices is 2 arcseconds.

maximal intensity of RHESSI image. The CLEAN image reconstruction method (Hurford et al. 2002) is applied to produce the RHESSI source, and the selected time interval for imaging is close to from 03:24:30 to 03:25:30 UT, which is close to the HXR peak time. Combining observations of magnetic field, EUV, SXR and HXR images, we can see that the source region of the eruption is located in a region of about $25 \times 50 \operatorname{arcsec}^{2}$ with mixed magnetic polarities. Blue arrows in Figure 2 (d), (e) and (i) have the same coordinates. The upper arrow indicates the fixed footpoint of a coronal loop. As time elapses, the footpoint becomes brighter and brighter. We infer that the accelerated electrons were released from the flaring region, transported along the magnetic field lines indicated by the coronal loop structure, and deposited energy at the other footpoint. The lower arrow is to mark the near top of the loop, and the change of the stand-off distance between the loop top and the arrow shows the height evolution of the loop top. Yellow arrows in Figure 2 (d) and (e) indicate the position of the HC front.

Figure 2 (f-i) are synthetic images with AIA $94 \AA$ basedifference images in green channel and AIA $211 \AA$ basedifference images in red channel observed from 03:24 UT to 03:26 UT. Figure 2 (i) displays the synthesized image at the GOES peak time. Both a bubble-like $\mathrm{HC}$ bounded by a green curve and a bright front preceding the $\mathrm{HC}$ bounded by a white curve can be clearly seen. Following the evolution from Figure 2 (f) to (i), we can observe the northward deflection of $\mathrm{HC}$ which is further validated by the LASCO image in Section 3.4.

\section{ANALYSIS AND RESULTS}

\subsection{Propagation and Expansion of the Hot Channel}

In order to display the rising motion of the $\mathrm{HC}$ nose and flank as well as the lateral expansion of the HC flank, we select two slices (1 and 2) along its propagation direction as shown in Figure 3 (a), and three slices 4 to 6 along the expansion direction of the HC flank which is perpendicular to the main propagation direction (slice 1) as shown in Figure 3 (b). Slice 3 is selected to track the $\mathrm{HC}$ deflection. In the minutes after $\sim 03: 26: 30 \mathrm{UT}$, the change of the HC's direction takes place along slice 3 in Figure 3 (a). The width of the slices is $\sim 2$ arcseconds (3 pixels) except for slice 3 ( of which the width is $\sim 7$ arcseconds (12 pixels)). The kinematic evolution of the $\mathrm{HC}$ along slices 1 to 3 are shown in Figure 4. In Figure 4 (a), to create the distance-time plots along slices 1 to 3 , we stack the base difference intensity along each slice with time. The position of the HC front along slices 1 to 3 is marked by blue, yellow, and orange plus signs, respectively. The track marked by the white arrow in the upper panel indicates the coronal loop introduced in Figure 2. Although the signal-to-noise ratio along slice 3 is very low, we can still observe the appearance of the signal later after 03:26:30 UT. One of the interpretations may be the possible northward deflection of the HC. Fit- 

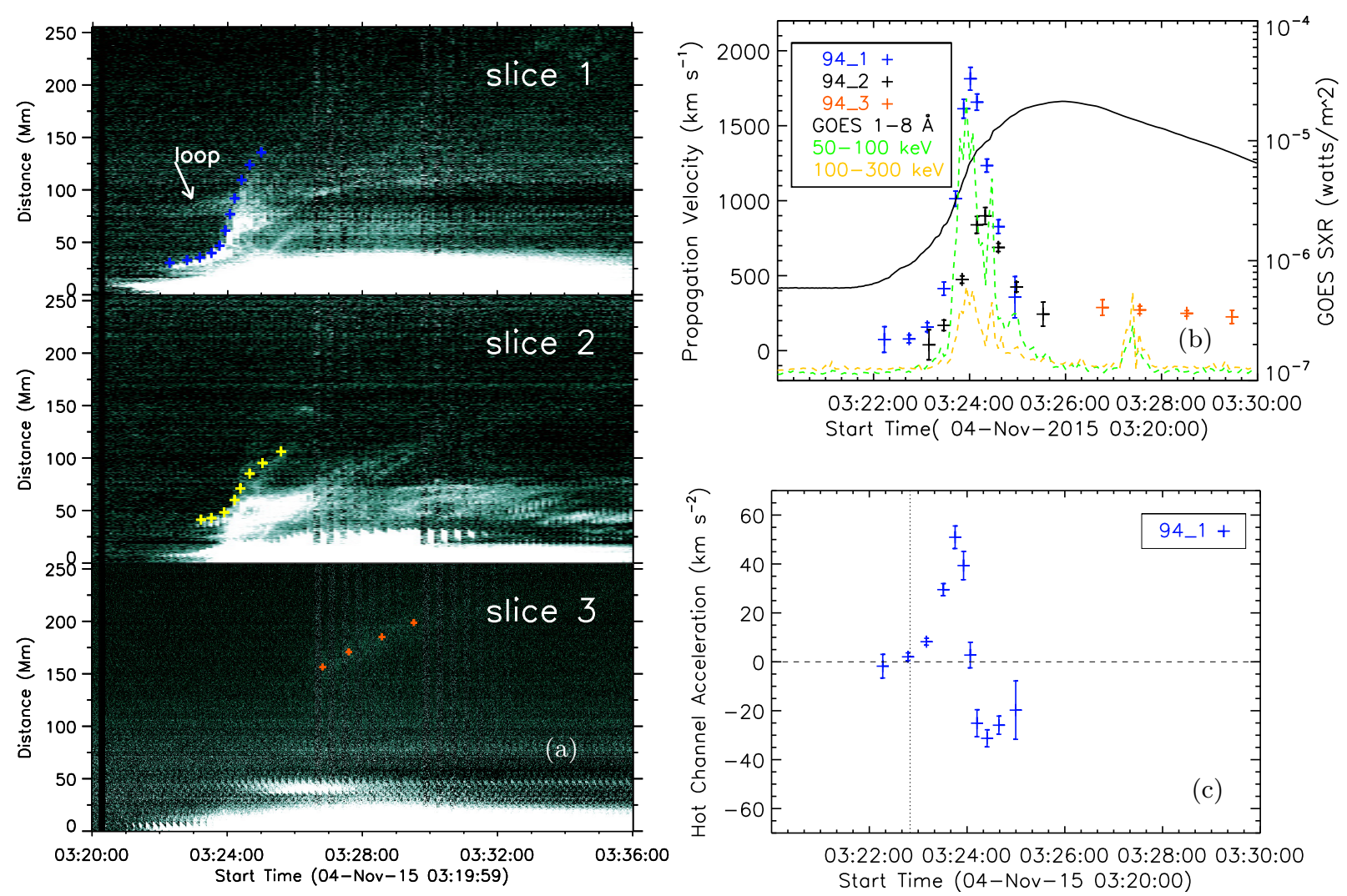

Figure 4: (a) Base difference distance-time plots of the $94 \AA$ passband along the slices 1,2 and 3 as shown in Figure 3 (a). The track marked by the white arrow denotes the distance evolution of the coronal loop. (b) Velocity evolution of the HC along slices 1, 2, and 3. The black-solid profile denotes the GOSE 1-8 $\AA$ SXR flux of the associated flare, and the yellow (green) curve is the photon count rates in the high energy band of 100-300 (50-100) keV. (c) The evolution of acceleration along slice 1 . The vertical dotted lines indicate the transit time at $\sim 03: 22: 50$ UT between the slow rise and fast rise phases. The error bars along slices 1 and 2 (slice 3) in panels (b) and (c) are propagated from the uncertainty of $\pm 6(12) \mathrm{Mm}$ in the distance measurements.

ting the distance-time data points with smoothing cubic splines, we further derive reliable velocities and accelerations. The deduced velocity and acceleration data points are presented in Figure 4 (b) and (c). Concerning the error analyses of the kinematics, we estimate that the maximal uncertainty in the distance measurement along the track of $\mathrm{HC}$ front is about $6 \mathrm{Mm}(12 \mathrm{Mm})$ for slices 1 and 2 (slice 3 ) by randomly tracing the front for a few times. Thus, we assume the distance uncertainty is $6 \mathrm{Mm}(12 \mathrm{Mm})$ above and below each point along the track for slices 1 and 2 (slice 3 ). Then this uncertainty is propagated to the uncertainties in velocity and acceleration with 100 Monto Carlo simulations. In such simulations, 100 randomly selected distance values following a Gaussian distribution with $3 \sigma=6 \mathrm{Mm}$ (12 Mm along slice 3 ) are used at each distance-time data point. Then the velocity and acceleration are derived 100 times using these randomly distributed distance values with the same procedure described above. The derived error bars are indicated in Figure 4 (b) and (c).
Figure 4 reveals that the kinematics of the $\mathrm{HC}$ along its main propagation direction (slice 1) can be clearly divided into three phases: a slow rise phase before 03:22:50 UT with small velocity and acceleration, a following fast rise phase from 03:22:50 UT to 03:24:10 UT with a peak velocity of about $1800 \mathrm{~km} \mathrm{~s}^{-1}$ and a peak acceleration of about $50 \mathrm{~km} \mathrm{~s}^{-2}$. The second phase is the main acceleration phase. The third phase after 03:24:10 UT is the residual acceleration phase and is characterized by a peak deceleration of about $30 \mathrm{~km} \mathrm{~s}^{-2}$ (more details can see Table 1 ).

Figure 4 (b) demonstrates that the HC main acceleration phase coincides very well with the flare impulsive phase as indicated by the RHESSI light curves. It implies that the impulsive acceleration phase of the $\mathrm{HC}$ is closely related to the magnetic reconnection process. This scenario is in general consistent with the CME-flare temporal relationship described in Zhang et al. (2001) and Zhang \& Dere (2006). However, there are a few particularities we need to point out for this event. 

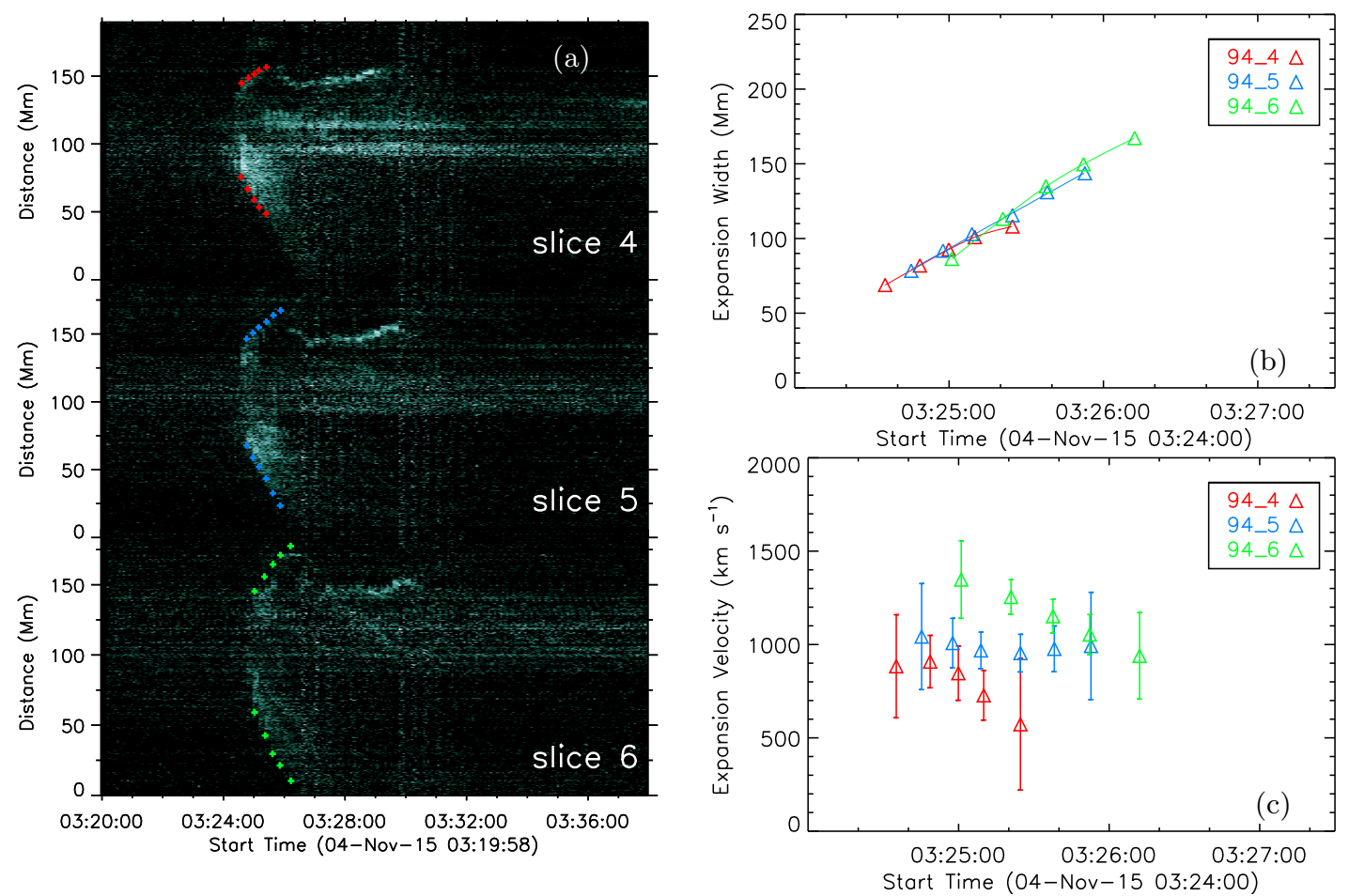

Figure 5: (a) Base difference distance-time plots of the $94 \AA$ passband along slices 4-6 as shown in Figure 3 (b). (b) The evolution of the HC width along slices 4-6 with different colors obtained from the data in panel (a). (c) The evolution of the HC expansion velocity derived from the data in panel (b). The error bars in panel (c) are propagated from the uncertainty of $\pm 6 \mathrm{Mm}$ in the distance measurements.

Table 1: Different phases of the HC \& the bright front (BF) along slice1

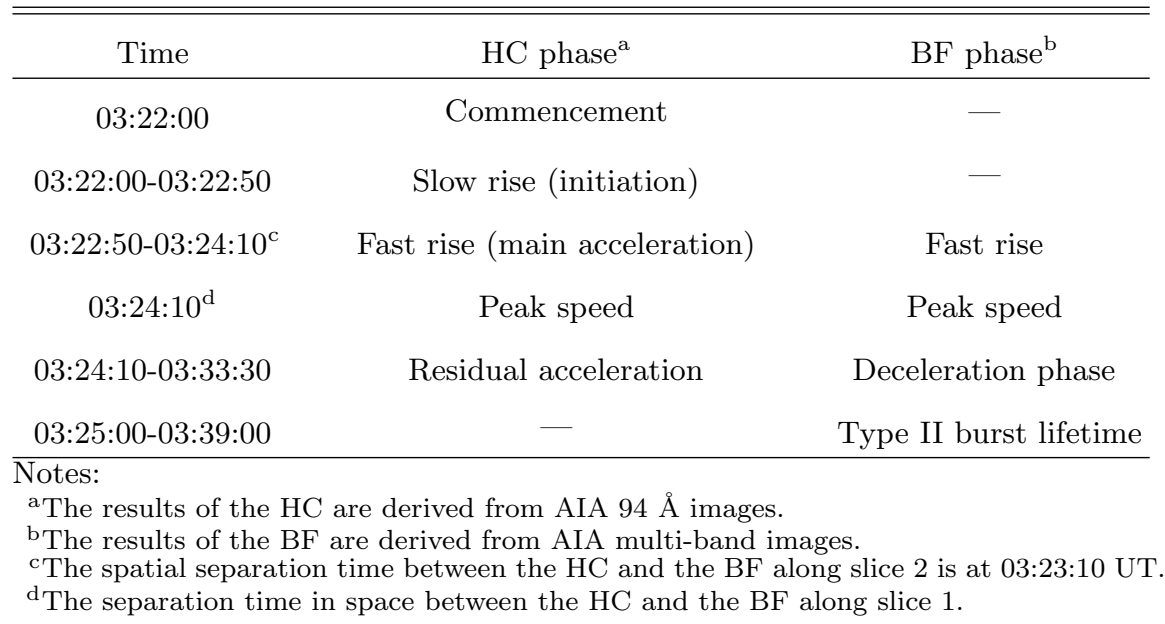

- The maximal acceleration rate of about $50 \mathrm{~km} \mathrm{~s}^{-2}$ during the main acceleration phase is two orders of magnitude larger than the average rate of $331 \mathrm{~m}$ $\mathrm{s}^{-2}$ given in Zhang \& Dere (2006) and one order of magnitude larger than their maximal value of 4464 $\mathrm{m} \mathrm{s}^{-2}$.

- The duration of the main acceleration phase is no more than two minutes, which is smaller than the minimal value of 6 minutes in Zhang \& Dere (2006).
- The acceleration magnitude (A) and the duration $(\mathrm{T})$ is in general following the rule proposed by Zhang \& Dere (2006) that the shorter the duration, the larger the acceleration. However, the magnitude A of $50 \mathrm{~km} \mathrm{~s}^{-2}$ is higher than the value derived from the scaling law $\mathrm{A}\left(\mathrm{m} \mathrm{s}^{-2}\right)=10,000 \mathrm{~T}^{-1}$ (minute) derived in Zhang \& Dere (2006).

- The deceleration of the $\mathrm{HC}$ in the residual accel- 
eration phase with maximal value of about $30 \mathrm{~km}$ $\mathrm{s}^{-2}$ is much larger than the reported value of 131 $\mathrm{m} \mathrm{s}^{-2}$ in Zhang \& Dere (2006).

Figure 5 presents the lateral expansion properties of the HC flank. Figure 5 (a) includes three stack plots of the AIA $94 \AA$ base-difference intensity along slices 46. The derived $\mathrm{HC}$ widths along these three slices are demonstrated in Figure 5 (b) in different colors. The corresponding expansion velocities and their associated error bars are calculated with the same method as used for the propagation. Figure 5 (c) reveals that the expansion velocity has a peak value of about $1350 \mathrm{~km} \mathrm{~s}^{-1}$ along slice 6 at 03:25:00 UT, and slows down to about $1000 \mathrm{~km} \mathrm{~s}^{-1}$.

In this event, the duration of the M-class flare impulsive phase is only about four minutes. The extreme fast speed and acceleration could be a combination effect of the radial motion of $\mathrm{HC}$ geometric center and the expansion of the $\mathrm{HC}$ front in all directions. The expansion of the $\mathrm{HC}$ front could be caused by the magnetic reconnection that adds new flux surrounding the existing component of the FR. In addition, the release of a good amount of energy in a very short time might also be the reason of the rather high value of deceleration. The deceleration is caused by the constraint of the magnetic field lines overlying the $\mathrm{HC}$ as will be see in Section 3.4. We find that the $\mathrm{HC}$ reached a distance of about $1.1 \mathrm{R}_{\odot}$ when it attained the peak velocity at 03:24:10 UT in AIA FOV. In Zhang \& Dere (2006), the analyses were based on the observations with LASCO C1, C2, and $\mathrm{C} 3$ whose FOV is from $1.1 \mathrm{R}_{\odot}$. Therefore, our event can be regarded as a complementary case for the statistics of the CME kinematics. However, when comparing our event with some other events with the peak velocity also occurred below $1.1 \mathrm{R}_{\odot}$ (e.g. Cheng et al. 2014), the characteristics of its high peak velocity, acceleration and deceleration, and short energy release is still very prominent. Moreover, due to its very high velocity, although the deceleration is also very high, the $\mathrm{HC}$ was able to reach the LASCO C2 FOV and did not evolve to a failed-eruption event as in Cheng et al. (2014) and Song et al. (2014).

\subsection{Piston-driven Shock Wave}

\subsubsection{Radio Type II Burst}

Type II solar radio bursts are slowly drifting structures identified in dynamic spectra. They are generally considered to be radio signatures of shock waves. Electrons accelerated at the shock front generate Langmuir waves which are converted to electromagnetic waves near the electron plasma frequency $f_{p}$ and $2 f_{p}$. Type II emission bands are sometimes showing split to two lanes of similar intensity and morphology, so called band split. This characteristic is frequently consider to be due to emission from the upstream and downstream shock region (Smerd et al. 1974, 1975). In our event, the metric type II burst, observed by the Learmonth and Culgoora radio spectrographs, exhibits a well defined fundamental and a second-harmonic emission band (hereinafter F- and $\mathrm{H}$-band, respectively), both being split in two parallel lanes. To obtain complete F- and H-bands, we combine the dynamic spectrum observed by the Learmonth radio spectrograph in the frequency range of $25-180 \mathrm{MHz}$, and the dynamic spectrum observed by the Culgoora radio spectrograph in the frequency range of 18-25 and 180-1000 MHz. To further make the F- and H-bands prominent, we reduce the noise level. The data points in the radio-quiet time are selected as the background level of radio emission in time intervals 03:12-03:20 UT and 03:42-03:48 UT. For each frequency, we obtain a mean value of the background from the data in the chosen time ranges. Then, the intensity in each frequency is divided by the obtained mean value correspondingly. The final spectrum is presented in the upper panel of Figure 6 . The type II burst signals commence at about 03:25:00 UT with an unusual higher starting frequency of about $320 \mathrm{MHz}$ in the F-band, which is higher than $215 \mathrm{MHz}$ derived in Kumari et al. (2017) for the same event. The band-split structures are also visible which were not analyzed in Kumari et al. (2017). The distinguished F-and $\mathrm{H}$-band emissions are marked by red pluses and black asterisks, respectively. The segments with clear band-split signals are indicated by vertical dashed lines. We find that the lower (higher) H-band frequency $f_{H L}\left(f_{H U}\right)$ is proportional to the lower (higher) F-band frequency $f_{F L}$ $\left(f_{F U}\right)$ with $f_{H L} \simeq 1.93 f_{F L}\left(f_{H U} \simeq 1.93 f_{F U}\right)$.

On account of the longer lifetime of the lower branch of the H-band, we utilize $f_{H L}$ to derive the upstream plasma frequency with $f_{p} \simeq f_{H L} / 1.93$, and further obtain the electron density $n$ in units of $\mathrm{cm}^{-3}$ with $f_{p}=$ $9 \times 10^{-3} n^{\frac{1}{2}} \mathrm{MHz}$. To convert the derived electron density to a radial distance, a density model $n(r)$ has to be invoked. In our case, the hybrid model proposed by Vršnak et al. (2004) is used, and it has a smooth transition from the active region corona to the interplanetary range:

$$
n_{\left[10^{8} \mathrm{~cm}^{-3}\right]}=\frac{15.45}{R^{16}}+\frac{3.16}{R^{6}}+\frac{1}{R^{4}}+\frac{0.0033}{R^{2}} .
$$

The inverted radial distances are illustrated by the red squares in Figure 7. We can see that the Type II source formed at a low height below $1.1 \mathrm{R}_{\odot}$. The red solid line is the corresponding cubic spline fitting. we have derived the velocity-time plot which is shown by blue squares in Figure 7. The associated error bars are propagated from the uncertainty in frequency of $5 \%$. The motion of the type II source slows down from $1750 \mathrm{~km} \mathrm{~s}^{-1}$ at 03:25 UT to $500 \mathrm{~km} \mathrm{~s}^{-1}$ at 03:38 UT.

Band split in radio dynamic spectra is useful to infer 


\section{-Learmonth--Culgoora-}
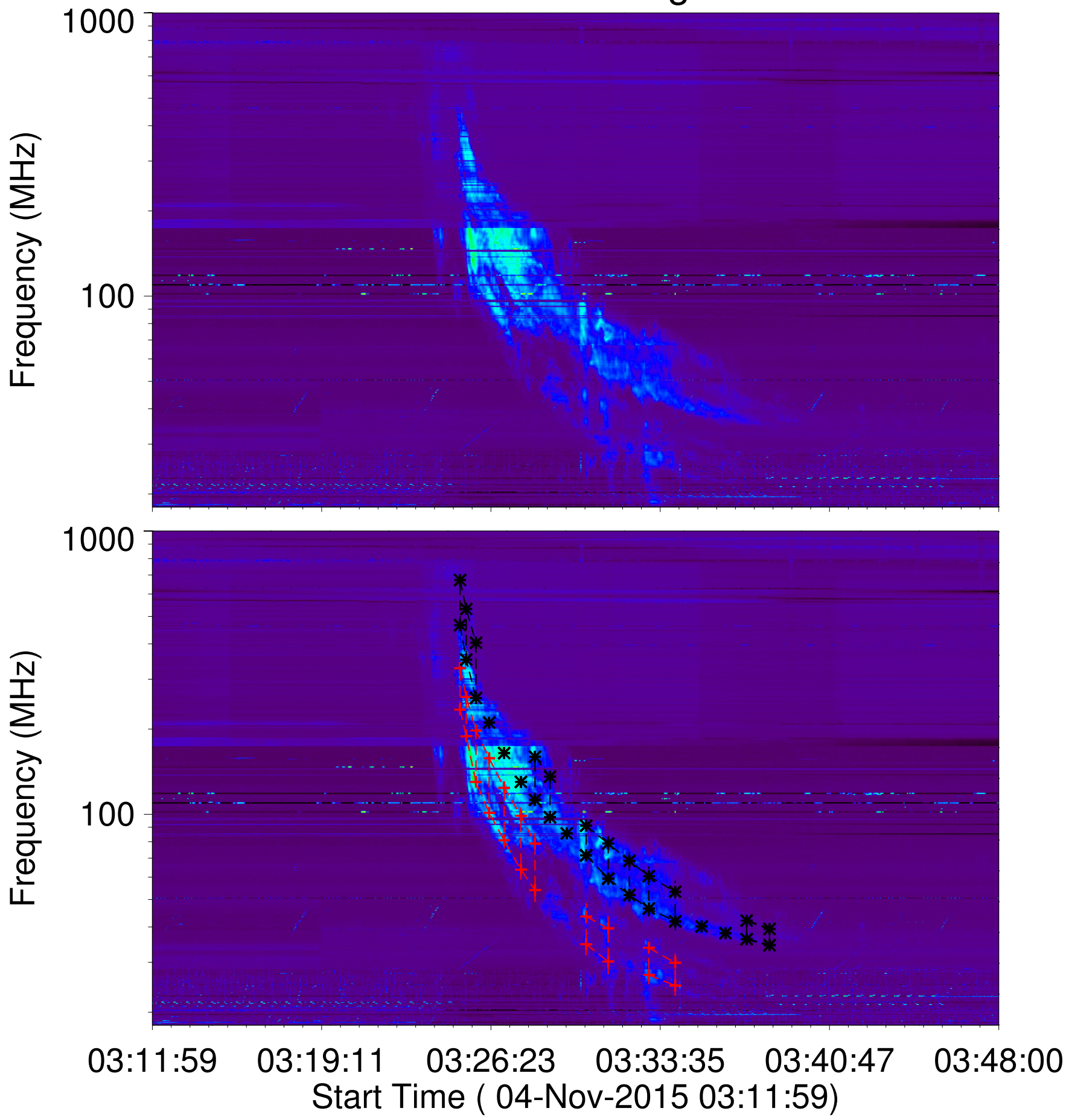

Figure 6: Radio dynamic spectrum from the Learmonth and Culgoora radio spectragraph. The spectrum in the frequency range from 25 to $180 \mathrm{MHz}$ is observed by Learmonth spectrograph, while the spectrum in the range of 18-25 $\mathrm{MHz}$ and $180-1000 \mathrm{MHz}$ is by Culgoora spectrograph. The bottom panel is the same as the top panel except for the marking symbols. In the bottom panel, the data points with visible band split signals are marked by the dashed lines. Red plus and black asterisk signs delineate the $\mathrm{F}$ - and $\mathrm{H}$-band, respectively. 


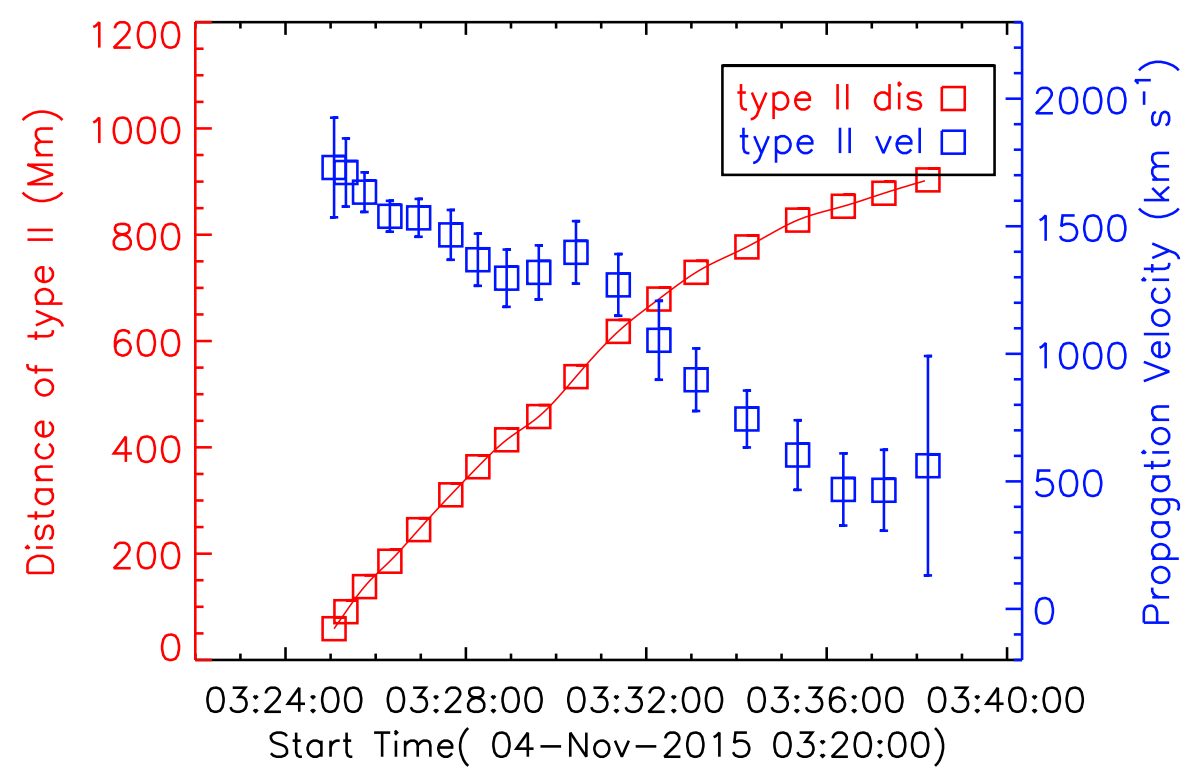

Figure 7: Properties of the type II bursts. The red squares represent the inverted radial distances of the type II burst using the density model of Vršnak et al. (2004). The blue square signs denote the shock radial speed derived from the type II radio burst.

shock wave properties (Vršnak et al. 2001, 2002, 2004; Gopalswamy et al. 2012; Su et al. 2016). The instantaneous split $\Delta f(t)$ at the moment $t$ is usually defined as the difference $\Delta f(t)=f_{U}(t)-f_{L}(t)$, where $f_{U}\left(f_{L}\right)$ is the frequency along the upper (lower) frequency branch. Then the relative instantaneous split is the ratio of $\Delta f(t) / f_{L}(t)$ which spans in a range from 0.14 to 0.48 . The derived relative split in our event is within the range of 0.05-0.6 obtained by Vršnak et al. (2001). The compression ratio $X$ is defined as the shock down-stream to up-stream density ratio $n_{2} / n_{1}$, and is a quantity which characterizes the shock strength. In Figure 8 (a), the compression ratio, deduced with $n_{2} / n_{1}=\left(f_{F U} / f_{F L}\right)^{2}$ and $n_{2} / n_{1}=\left(f_{H U} / f_{H L}\right)^{2}$ showing red and black signs represent $X$ derived from the band split of $\mathrm{F}$ - and $\mathrm{H}$ band, respectively. We find that the shock strength decreases from 2.2 to 1.3 within 14 minutes. To compute the Alfvénic mach number $M$, we assume a quasiperpendicular shock and adopt the formula introduced in Draine \& McKee (1993),

$$
\frac{n_{2}}{n_{1}}=\frac{2(\gamma+1)}{\left\{D+\left[D^{2}+4(\gamma+1)(2-\gamma) M^{-2}\right]^{1 / 2}\right\}},
$$

where $D=(\gamma-1)+\left(2 / M_{s}^{2}+\gamma / M^{2}\right), \gamma$ is the adiabatic index and set as $4 / 3$ and $5 / 3$, and $M_{s}=V_{s h} / C_{s}$ is the sonic Mach number in which $V_{s h}$ is the radial speed of the shock presented in Figure $7, C_{s}$ is the sound speed. For $\mathrm{T}=1.5 \mathrm{MK}$ and $m=1.27 \times 1.673 \times 10^{-24} \mathrm{~g}$ the average particle mass in the corona (Aschwanden 2005), and according to the formula

$$
C_{s}=\sqrt{\frac{\gamma p}{\rho}}=\sqrt{\frac{\gamma R T}{M}}=\sqrt{\frac{\gamma k T}{m}},
$$

the sound speed is $C_{s}=114(127) \mathrm{km} \mathrm{s}^{-1}$ for $\gamma=4 / 3$ (5/3). The Alfvénic Mach number $M$ which can be derived from Equation (2) is shown in Figure 8 (b). The primary uncertainty in $M$ is propagated from the compression ratio $X$. The calculated $M$ values are indicated by diamonds (triangles) with $\gamma=4 / 3(5 / 3)$. In Figure 8(c), $V_{A}=V_{s h} / M$ yields the Alfvén speed decreasing with the heliocentric distance. Once we get $V_{A}$, the magnetic field strength in the upstream medium can be calculated:

$$
B=5.1 \times 10^{-5} V_{A} f_{p} .
$$

The upstream frequency $f_{p}$ has been obtained thereinbefore, and shown in Figure 8 (d). Dulk \& McLean (1978) derived an analytical equation for $B(r)$ :

$$
B=0.5(r-1)^{-1.5},
$$

where $r$ is the heliocentric distance in units of solar radii. For $r\left(R_{\odot}\right)=1.3-2.1$, our measurements are somewhat higher, but comparable with the value given by this formula.

\subsubsection{Imaging Observations}

As indicated in Figure 2, a bright front appeared preceding the HC. Figure 9 shows the evolution of the EUV bright front in AIA multiple wavelengths. The top images of Figure 9 is observed at 03:25 UT at which the shock signal first appeared in radio dynamic spectra. The 

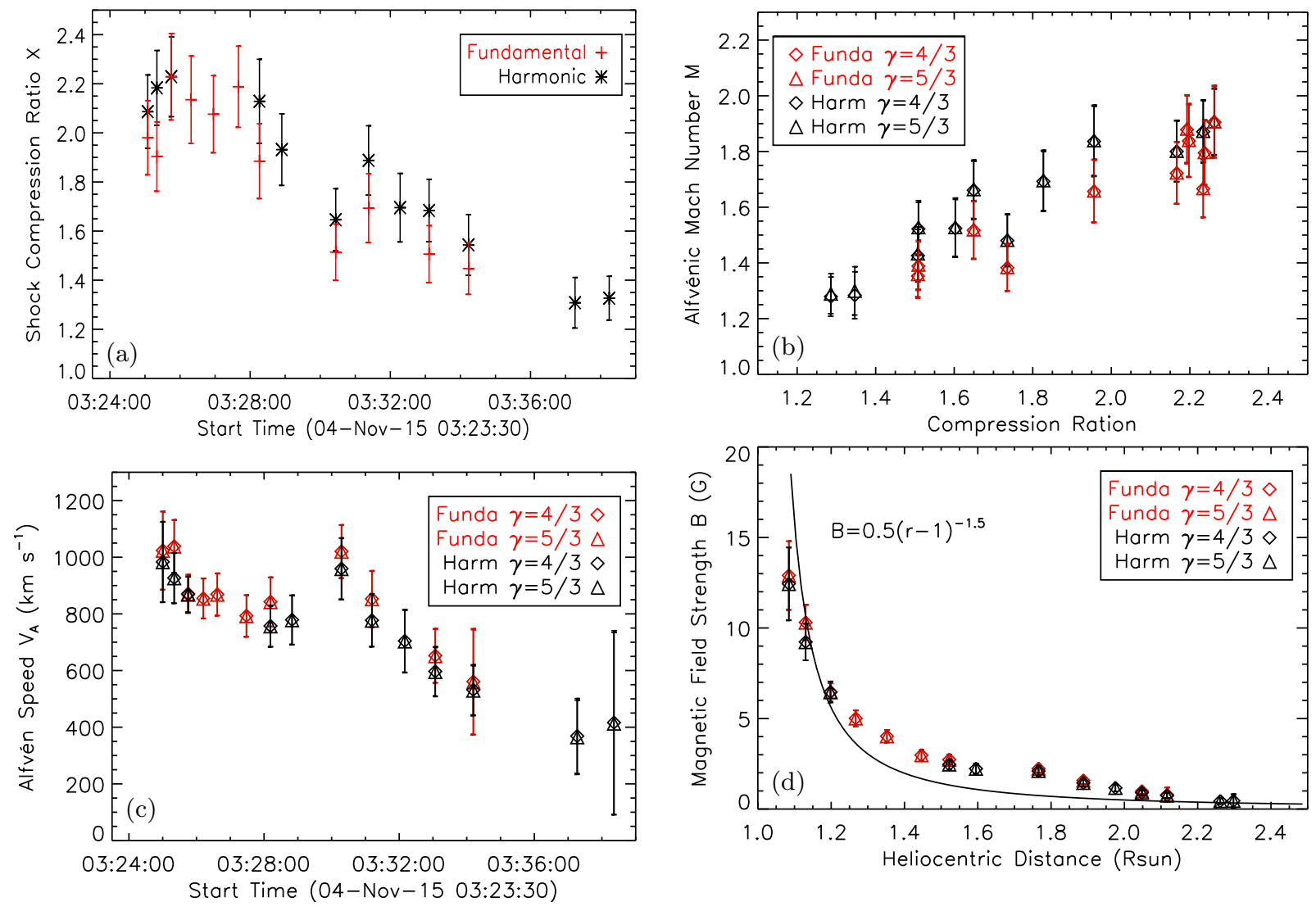

Figure 8: (a) Shock compression ratio $X$ calculated from the band split along the F-band (red plus signs) and the H-band (black asterisks). The color code in panels (b)-(d) follow the same denotation. (b) Alfvénic Mach number $M$ derived with the method of Draine \& McKee (1993) for $\gamma=4 / 3$ (diamonds) and 5/3 (triangles). (c) Alfvén velocity $V_{A}$ derived from the ratio of the shock radial speed $V_{s h}$ to the Alfvénic Mach number $M$. (d) Coronal magnetic-field strength $B$ obtained from $V_{A}$ and $f_{p}$. The solid line is the profile derived from the empirical formula in Dulk \& McLean (1978). The error bars in all panels are derived and propagated under the assumption of $5 \%$ uncertainty in frequency.

bright front is clearly seen in AIA $193 \AA$ and $211 \AA$ images, and only part of it can be distinguished in AIA 304 $\AA$ image at $~ 03: 26: 40$ UT. Figure 10 (a) is the composite of the tri-color AIA images in $193 \AA$ (green channel), 211 $\AA$ (red channel) and $304 \AA$ (blue channel) along slices in Figure 9 (a). Note that due to lack of the bright front signature in AIA $304 \AA$ along slice 1, the first panel in Figure 10 (a) is a bi-color image in AIA $193 \AA$ and $211 \AA$. Here, we regard the direction along slice 1 as the nose direction. The distance and derived velocities along slices 1, 2 and 7 are shown in Figure 10 (a) and (b). As indicated in Figure 10 (b), the bright front experiences an acceleration phase and reaches a maximal velocity of about $1900 \mathrm{~km} \mathrm{~s}^{-1}$ at 03:24:10 UT and then decelerates. The vertical dashed line indicates the time when the bright front separates from the HC flank, while the dotted line denotes the separation between the $\mathrm{HC}$ nose and the bright front in space. At 03:25 UT, although the speed of the bright front decreases to around $1200 \mathrm{~km}$ $\mathrm{s}^{-1}$, it still reaches over the local Alfvén speed shown in Figure 8 (c). In Kumari et al. (2017) the Gauribidanur RAdioheliograPH (GRAPH) observations revealed that the type II source at 03:31 UT was located immediately above the EUV bright front observed at 03:26 UT. Therefore, in combination of the GRAPH source location, analyses of the type II burst in radio dynamic spectra, and EUV bright front evolution, we tend to believe that these bright fronts have close relationship with the shock fronts both in time and in space. At least after 03:25 UT we can say that the bright fronts have gone through the nonlinear evolution and transformed into a shock wave. Before 03:25 UT the bright fronts may simply be an EUV wave front.

In Figure 11 (a), the inverted radial distances from the type II radio burst are illustrated by the green squares, together with the bright front distance marked by the blue asterisks, which are traced in the AIA composite image along the direction of the nose. The blue and green solid lines are the corresponding cubic spline fittings. In Figure 11 (b), the distinction between the veloc- 


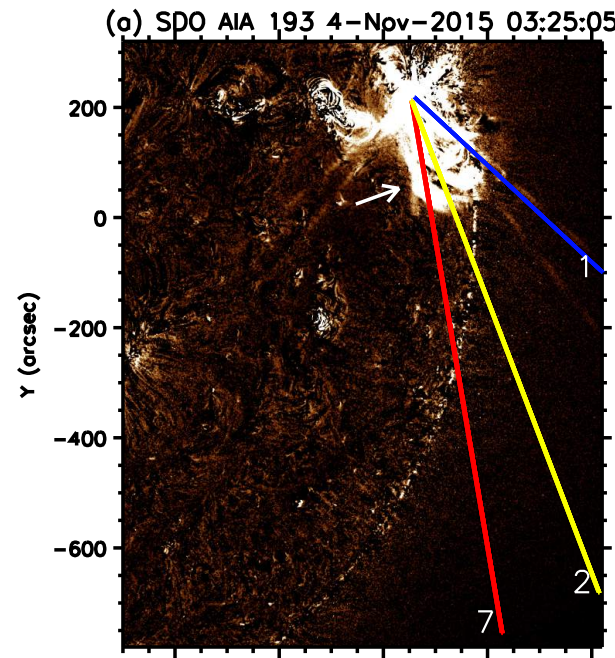

(b) SPO AIA 193 4-Nov-2015 03:26:41

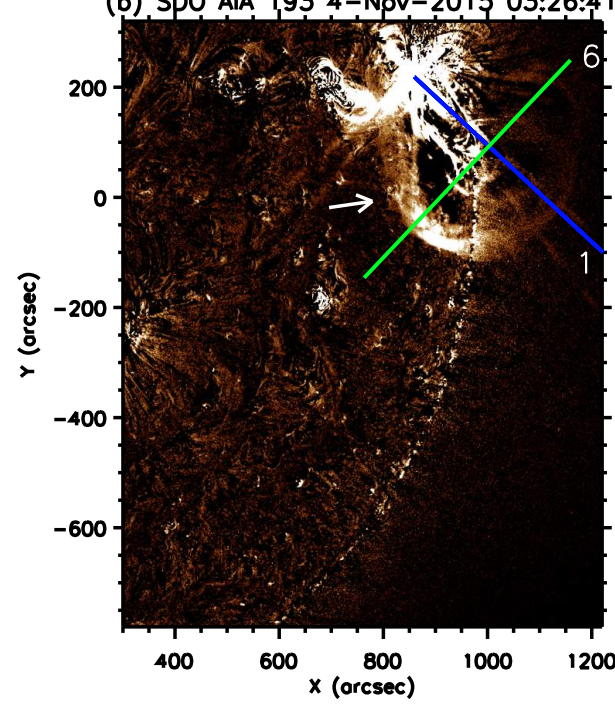

(c) SDO AIA 2,11 4-Nov-2015 03:25:01

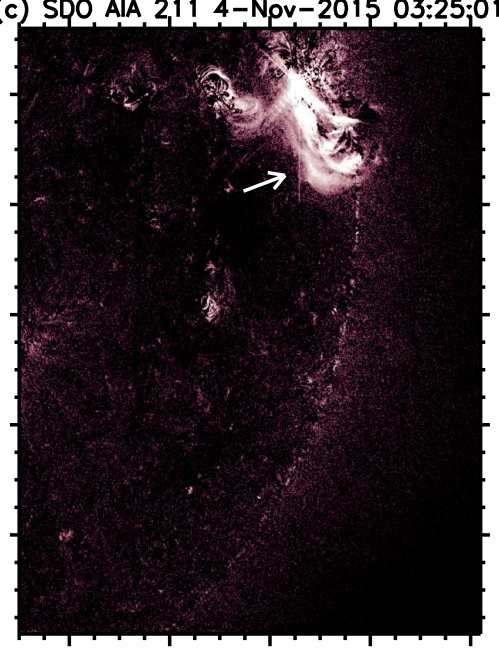

(e) SDO AIA 304 4-Nov-2015 03:25;06

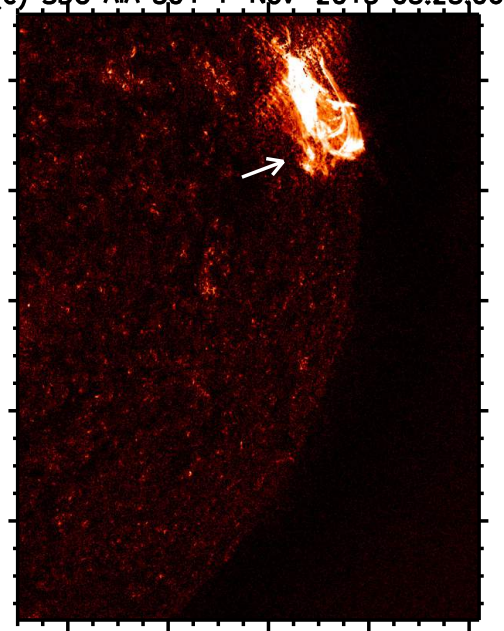

(d) SDO AIA 2,11 4-Nov-2015 03:26;36
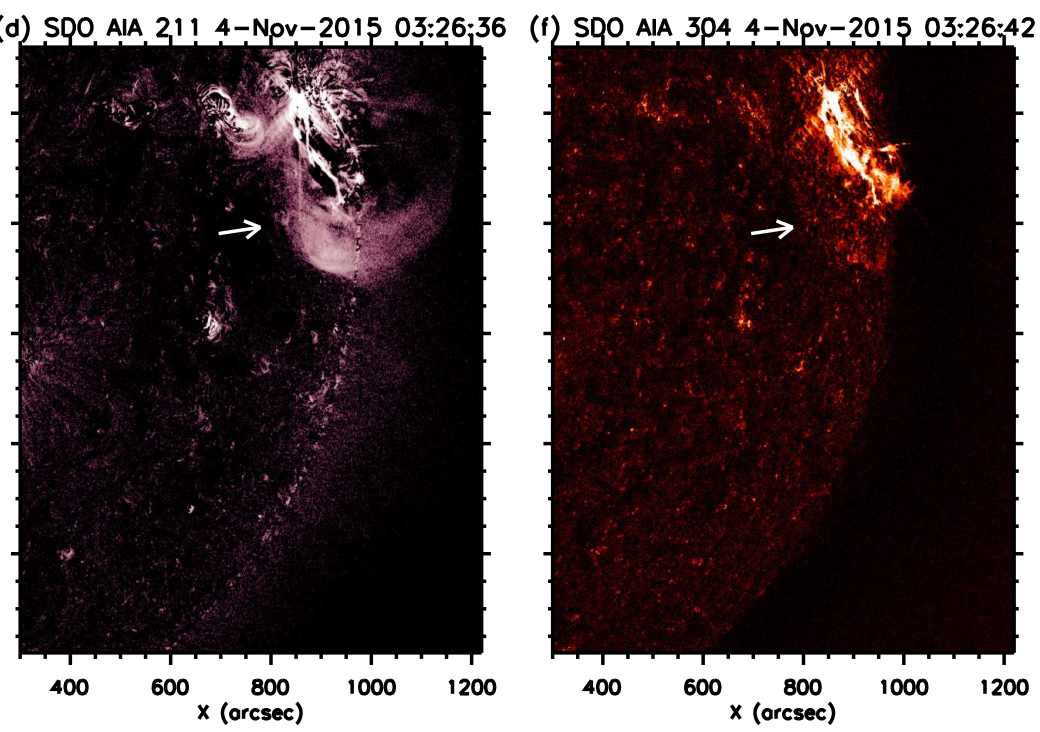

Figure 9: SDO/AIA base difference images in $193 \AA$ (left column), $211 \AA$ (middle column) and $304 \AA$ (right column) passbands. The time difference between the images in the first and second row is about 1.5 minutes (the time of each frame is indicated in the inset labels at the top of each panel). The slices in panel (a) numbered as 1 (in blue), 2 (in yellow), and 7 (in red) delineate the regions along which the shock kinematics is characterized (see Figure 10, panel a). In panel (b) the slices 1 and 6 mark the same region pointed by the homologous slices in Figure 3 but with extended lengths. The width of the slices is 2 arcseconds.

ity derived from type II frequency drift and the velocities from AIA imaging observations can be seen. Figure 11 (a) and (b) confirm non-radial propagation of the shock wave. Namely, the coronal electron density models describe the change of density in radial direction, and they are not very suitable for events with strongly non-radial propagation. For the type II source, the radial distances are calculated; while for the presumed shock in AIA images, the distances are measured along a given non-radial direction in the solar disk plane.

We have also investigated the stand-off distances between the $\mathrm{HC}$ and its driven shock along slices 1, 2 and 6 . Figure 12 (a-c) are composite distance-time images along these three slices with red, green, and blue channels in AIA $211 \AA, 94 \AA$, and $193 \AA$, respectively. The traced shock and $\mathrm{HC}$ fronts are denoted by white and yellow plus signs. The measured widths of the $\mathrm{HC}$ and the shock along slice 6 are indicated in. Figure 12 (d) with black triangles and asterisks, respectively. It can be found that the stand-off distance increases with time in this direction. The expansion velocities of the $\mathrm{HC}$ are slightly lower than the velocities of the shock as demonstrated by the orange triangles and asterisks. Figure 12 (e) includes the distance-time and velocity-time plots of the $\mathrm{HC}$ and the shock along the nose direction (slice 1) marked by blue and red symbols, respectively. The propagation ve- 

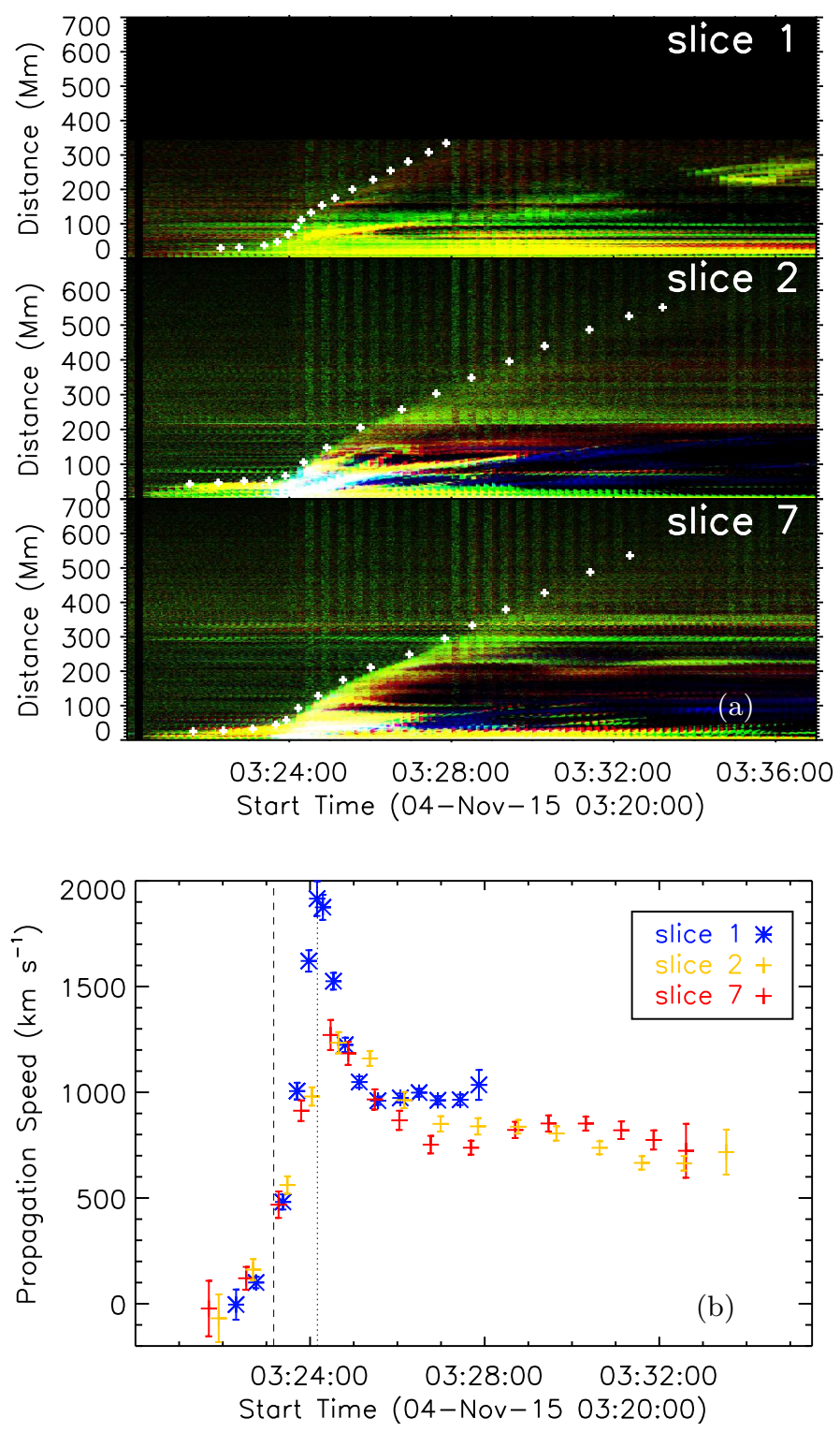

Figure 10: Top panel (a): Red-Green-Blue (RGB) representation of the synthesized base difference distance-time plots along the slices numbered as 1, 2, 7 in Figure 9 (a). The emission at $193 \AA, 211 \AA$, and $304 \AA$ are represented in the green, red, and blue channels, respectively. In the top panel, the composite distance-time plot only includes AIA 193 $\AA$ and $211 \AA$ images, as there is almost no signal along slice 1 in $304 \AA$ images. The plus signs in white color mark the tracked positions of the bright front. Bottom panel (b): Speed of the bright front along the slices 1, 2 and 7 (same color coding as in panel a of Figure 9; as derived from the tracks delineated by the white plus signs in the AIA tricolor frames shown in panel a). The vertical dashed and dotted line indicates the separation time between the HC and the EUV bright front along the flank and nose direction respectively shown in Figure 12. The error bars are propagated from the uncertainty of $\pm 6 \mathrm{Mm}$ in the distance measurements.

locity of the shock is also higher than the HC's velocity. The vertical dotted line indicates the time 03:24:10 UT, when the bright front and the HC started to separate in space. Figure 12 (f) reveals similar relation between the $\mathrm{HC}$ and the shock front along the flank direction (slice 2), and the separation time is $\sim 1$ minute earlier at 03:23:10 UT. We assume that the shock formation time is no later than the time when the shock signature was captured by radio spectra at around 03:25 UT. Thus, the formation time of the shock is less than 2 minutes after the onset of the $\mathrm{HC}$ main acceleration phase. Before 03:25 UT, the shock nose reaches a distance less than about 180 $\mathrm{Mm}$. The stand-off distance between the HC nose and the shock nose slightly increases with time.

The similarity in the overall evolution of the $\mathrm{HC}$ and the shock and the increase of the stand-off distance with 

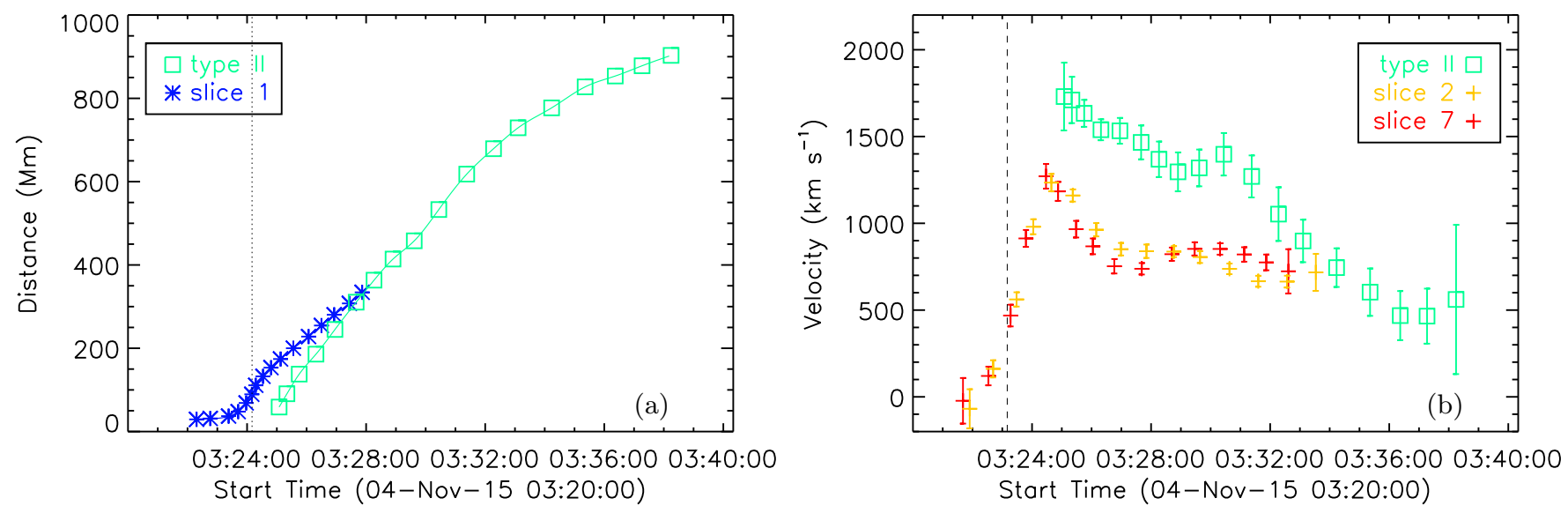

Figure 11: (a) Blue asterisks represent the bright front distance traced in the AIA tri-wavelength stack plot along slice 1. Green squares represent the inverted radial distances from type II burst using the density model of Vršnak et al. (2004). (b) Velocity evolution of the inferred shock. Green square signs denote the shock radial speed derived from the type II burst and plus signs represent the projected speeds of the shock in the AIA tri-wavelength image along slices 2 and 7. The vertical dotted (dashed) lines have the same meaning as shown in Figure 10. The error bars of the shock radial speed (green) are derived and propagated under the assumption of $5 \%$ uncertainty in frequency.

time fit to the piston-driven shock scenario (Warmuth 2015). A piston-driven shock is generated by the fast and/or impulsive expansion of a driver in all directions, like in an explosion. In our case, the fast and impulsive expansion of the $\mathrm{HC}$ drives the shock. During this driving phase, the shock wave propagation is controlled by the motion of the piston, and the stand-off distance between shock and piston increases. Once the piston decelerates, the shock detaches and can continue its propagation, although now without additional energy supply by the piston. The piston can be slower than the shock, and has to accelerate rapidly. Žic et al. (2008) found that the shock-formation time and shock-formation distance are approximately proportional to the acceleration phase duration of the piston, shorter for a higher source speed and acceleration. Therefore, the HC kinematics demonstrated in Figure 4 and Figure 5 can be used to interpret the short shock-formation time and low shock-formation height in our event.

\subsection{Thermal Properties of the Eruption}

We analyze the thermal properties of the $\mathrm{HC}$ and its piston-driven shock through the differential emission measure (DEM) method, which measures the contribution of plasma emission in a given temperature range. To reconstruct the DEM, we run the routine "XRT dem interative2.pro" in the Solar Software (SSW) package for each pixel in the region of interest. The code was originally developed by Weber et al. (2004) and has been modified slightly to work with AIA data (Cheng et al. 2012). Figure 13 (a) and (b) present the EM in two different temperature ranges for the $\mathrm{HC}$ driven shock and the HC. One can see that the shock region is primarily dominated by the plasma with $\log T=6.1-6.5$, and the $\mathrm{HC}$ is heated to a temperature of $\log T=6.8-7.2$. In Figure 13 (c) we plot the sum of the DEM curve of each pixel in the region shown in Figure 13 (a) and (b). Apparently, there are two peaks in the figure. The major contribution to the first peak at $\log T=6.2$ is primarily from the shock region. There may be also some contribution from the plasma in the surrounding regions. The second peak at $\log T=6.9$ corresponds to the HC structure. The thermal properties of the $\mathrm{HC}$ and its driven shock in our event are consistent with results obtained previously for large-scale events (e.g., Cheng et al. 2014; Su et al. 2015).

\subsection{CME in LASCO Field of View}

Due to the high speed of the $\mathrm{HC}$, it propagates to the LASCO/C2 FOV although suffering a significant deceleration. Figure 14 (a) and (b) show two snapshots during its propagation. Red dashed lines mark the leading edge of the CME. Its internal structure is quite diffuse and does not show the classical three components: core, cavity, and front. Probably the material in minifilaments involved in the eruption fell back to the surface so that they could not form a core in the LASCO FOV. We also notice that the propagation direction changes from southwest in the AIA FOV to equatorward in the LASCO FOV. The weak track, appearing in the distancetime plot along slice 3 in Figure 4 (a), also can reveal the deflection of the $\mathrm{HC}$ at the edge of the AIA FOV. It is presumably influenced by the overlying loops above the $\mathrm{HC}$ whose footpoints as marked by the red star in Fig- 

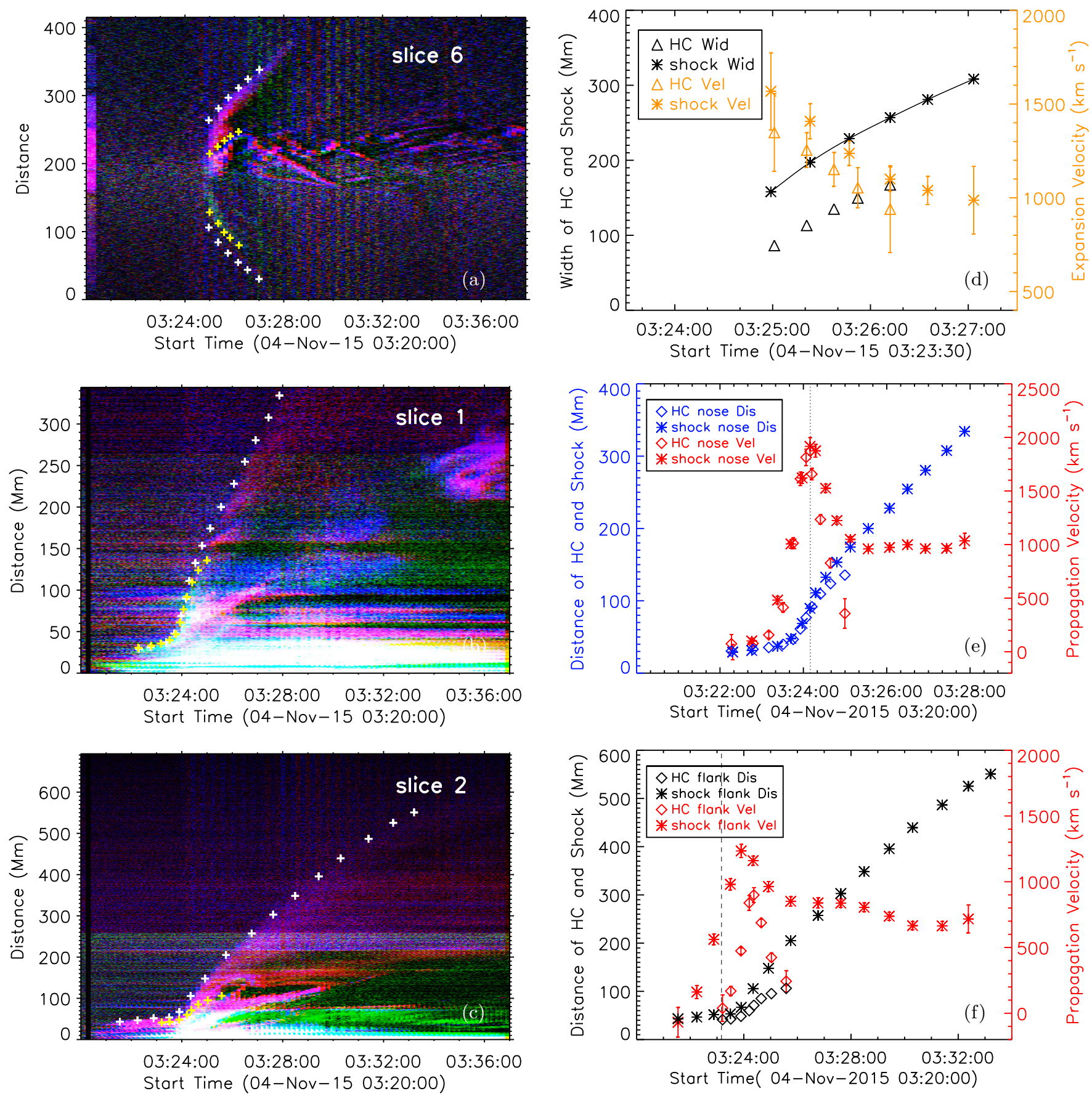

Figure 12: Panel (a): RGB representation of the synthesized running difference distance-time plot along slice 6 with the $211 \AA$ image in the red channel, the $193 \AA$ image in the blue channel and the $94 \AA$ image in the green channel. Panels (b)-(c): RGB representation of the synthesized base difference distance-time plots along slices 1 and 2 . In panels (a-c), the shock and HC fronts are denoted by white and yellow plus signs, respectively. Panel (d): Lateral expansion width of the HC (black triangles) and of its associated shock (black asterisks) along slice 6 (scale on the left axis), along with their corresponding lateral expansion velocity (in orange color, scale on the right axis) as derived from the smoothing cubic-spline fittings to the width-time data points. Panel (e): Distance-time (in blue color, scale on the left axis) and velocity-time (in red color, scale on the right axis) plots of the HC (diamonds) and the shock (asterisks) along slice 1. Panel (f): Evolution of the distance (scale on the left axis) and velocity (scale on the right axis) of both the HC and the shock along slice 2. The dotted (dashed) lines indicate the time when the shock and the $\mathrm{HC}$ along slice 1 (slice 2) start to separate in space. 

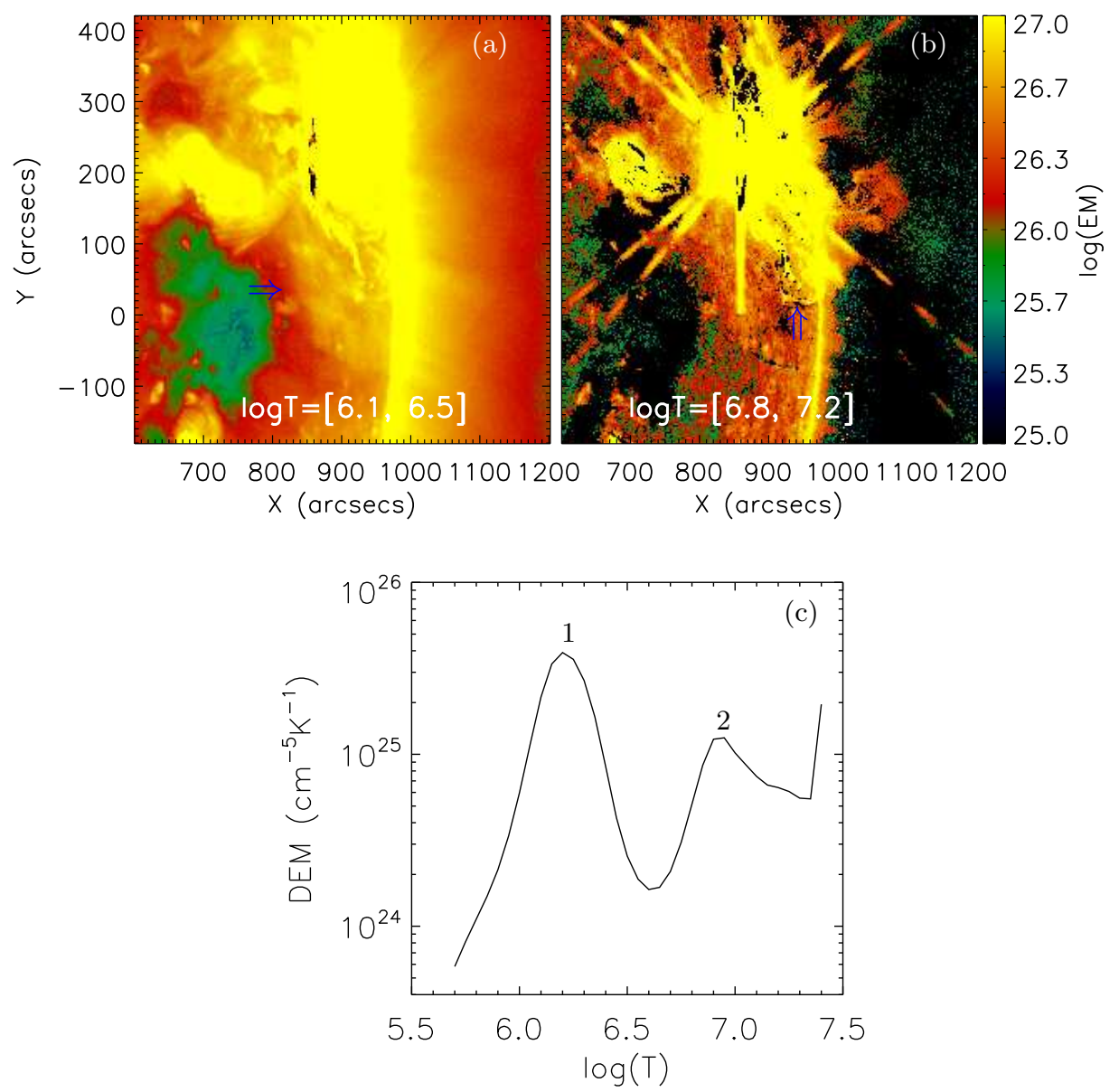

Figure 13: (a) EM in temperature range from $6.1-6.5$ in logarithm scale in which the presumed shock structure is best seen. The blue arrow points to the position of the shock region. (b) EM in the temperature range from $6.8-7.2$ in logarithm scale in which the hot channel is best seen. The blue arrow denotes the position of the HC. (c) The sum of the DEM curve of each pixel in the region shown in panels (a) and (b). The first peak corresponds to the contribution of the emission mainly from the shock compression region, while the second peak from the HC.

ure 14 (c). The magnetic field lines in Figure 14 (c) are derived from the Potential Field Source Surface (PFSS) method (Schrijver \& De Rosa 2003). The characteristics of the CME are presented in Figure 15. A linear fit to the height-time data in Figure 15 (a) yields an averaged velocity of $390 \mathrm{~km} \mathrm{~s}^{-1}$. To derive the CME mass, we assume that it propagates in the plane of the sky and calculate the electron column density and the resultant mass in each pixel according to the Thomson scattering theory (Feng et al. 2015a,b). The total mass is then derived by summing the mass in the area defined by the red dashed lines in Figure 14. We find that the CME mass increases with time and later reaches an almost constant value. It has a magnitude of $10^{14} \mathrm{~g}$, but is subject to a large uncertainty in the background subtraction. Because of the existence of a preceding CME indicated by the white arrow in Figure 14 (a), the mass calculated from basedifference images may be under-estimated. Therefore, we subtract two different backgrounds before mass calcula- tion. One is a 12-hour minimum image calculated as the minimum values of images from 00:45 UT to 12:57 UT, the other is a pre-event image at 03:45:09 UT. In Figure 15 (b), the corresponding mass is delineated by the dashed line in the former case, by the dash dotted line in the later case. Their average is indicated by the solid line. Based on the computed mass, height, and velocity, we estimate the potential and kinetic energy of the CME. The obtained results are illustrated in Figure 15 (c) and (d). The magnitude of the potential energy is on an order of $10^{30} \mathrm{erg}$, which is about one order of magnitude larger than the kinetic energy.

\section{DISCUSSIONS AND CONCLUSIONS}

We have made comprehensive study on the small-scale short-duration eruption associated with mini-filaments occurred on November 4th, 2015. The source region of the eruption is a small-compact area with multiple magnetic polarities located at the edge of NOAA AR 12445 

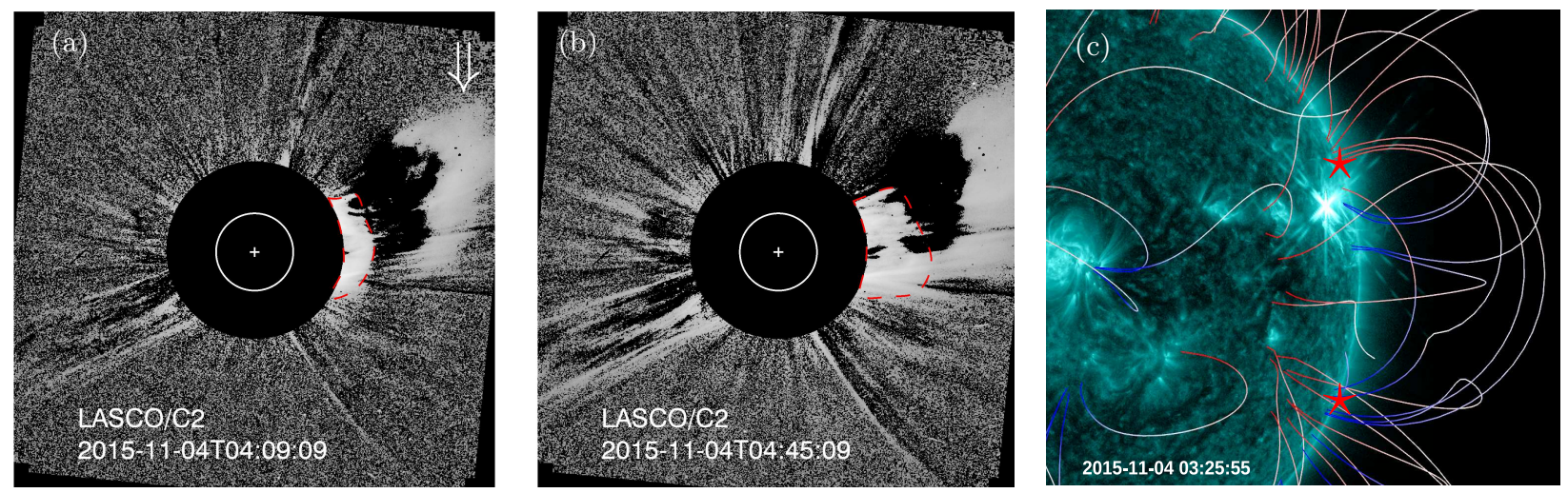

Figure 14: (a)-(b) White-Light coronagraph observations of the CME at different times. The red dashed line defines the leading front of the CME, and the white arrow points to a preceding CME event. The white circle and plus sign defines solar disk and solar center, respectively. (c) Coronal magnetic field lines extrapolated with the PFSS method over-plotted in the AIA $131 \AA$ image. The footpoints of the streamer arcades overlying the HC are marked by two red stars.

close to the west solar limb. Kumari et al. (2017) analyzed the same shock event. In our paper we focused not only on the analysis of the type II burst and shock, but also on the analysis of its driver, i.e., the CME. We did not use the shock to represent the near-Sun kinematics of the CME as in Kumari et al. (2017). We present detailed analyses of the CME hot channel itself and its temporary and spatial relationship with the shock. Through the study of HC-shock relationship, we then can infer why the shock has an unusual high starting frequency and low formation height which was not included in Kumari et al. (2017). The unusual large acceleration and high speed of the $\mathrm{HC}$ are the cause of high starting frequency. Concerning the type II dynamic spectra, we used higherresolution data in which band split can be observed which allows us to further infer important parameters related to the shock than Kumari et al. (2017). Therefore, the main conclusions are listed below:

- Although the kinematics of the $\mathrm{HC}$ is in general consistent with the scenario of three-phase evolution, it has a very short duration (less than 2 minutes) in the main acceleration phase, and has an exceptional high maximal acceleration rate $(\sim 50$ $\left.\mathrm{km} \mathrm{s}^{-2}\right)$, peak velocity $\left(\sim 1800 \mathrm{~km} \mathrm{~s}^{-1}\right)$, and maximal deceleration rate $\left(\sim 30 \mathrm{~km} \mathrm{~s}^{-2}\right)$, comparing to the kinematic statistics in Zhang \& Dere (2006). We also detect a very fast expansion along the $\mathrm{HC}$ flank with a peak velocity of about $\sim 1350 \mathrm{~km} \mathrm{~s}^{-1}$.

- The fast motion of the HC along different directions acts as a piston and drives a fast shock. The kinematic measurements including velocity, acceleration, stand-off distance, etc., of the $\mathrm{HC}$ and its driven shock fit the piston-driven shock scenario. The analyses of the associated type II burst to- gether with the AIA imaging data reveals very low starting height of the type II burst, at about $1.1 \mathrm{R}_{\odot}$. The type II band split is used to constrain the decrease of the shock compression ratio $(2.2$ to 1.3), Alfvénic mach number (1.9 to 1.3), from where the Alfvén speed (1000 to $400 \mathrm{~km} \mathrm{~s}^{-1}$ ) and the coronal magnetic field strength $(13$ to $0.5 \mathrm{G}$ ) are derived in the heliocentric distance from 1.1 to $2.3 \mathrm{R}_{\odot}$.

- The thermal properties of the $\mathrm{HC}$ and its driven shock are consistent with those derived for largescale events based on the DEM analyses.

- The CME observed in the LASCO/C2 FOV has a small leading-edge velocity $\left(390 \mathrm{~km} \mathrm{~s}^{-1}\right)$, small mass $\left(\sim 10^{14} \mathrm{~g}\right)$, potential $\left(\sim 10^{30} \mathrm{erg}\right)$ and kinetic $\left(\sim 10^{29} \mathrm{erg}\right)$ energy.

Concerning flare and CME energetics, Emslie et al. (2012) and Feng et al. (2013) have found the energy equal-partition between the flare and the CME for largescale eruptions. For this small-scale event, the total radiated output classified as the 'final' energy (Emslie et al. 2005 ) is used to estimate the flare energy, and the sum of the potential and kinetic energy is a measure of the CME energy. The GOES and RHESSI observations show impulsive and short duration M1.9 flare, shorter than that of those large-scale eruptions which often last for tens of minutes to several hours in previous studies (Chamberlin et al. 2012; Feng et al. 2013; Cheng et al. 2014, 2015). Chamberlin et al. (2012) found that the total radiated output of flares depends more on the flare duration than the typical GOES X-ray peak magnitude classification. Here, we estimate the output irradiance by using the data of the EUV Variability Experiment (EVE; Woods et al. 

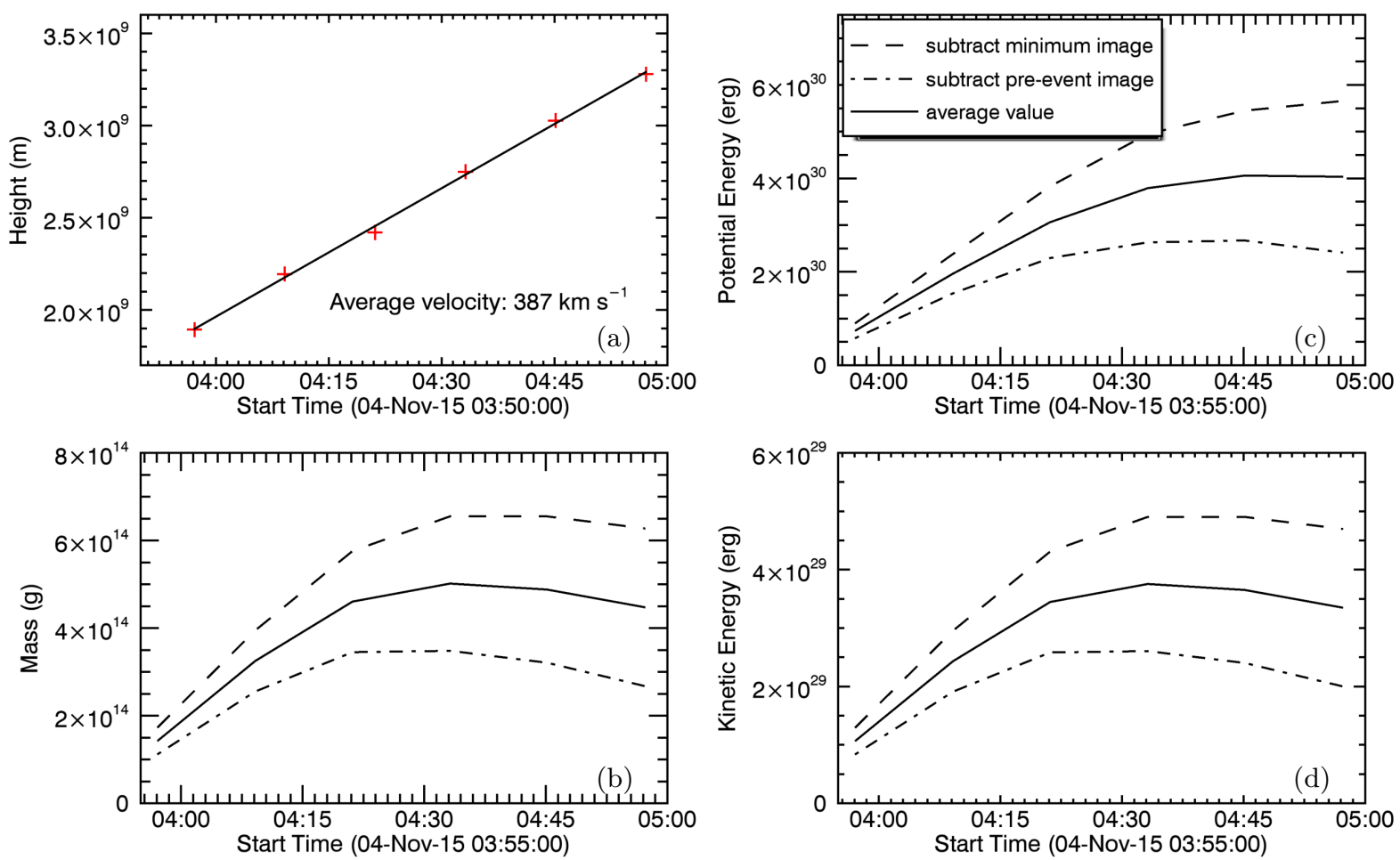

Figure 15: Panel (a): Height-time plot of the CME leading edge. Panels (b)-(d): Mass, potential energy, and kinetic energy profiles, respectively, of the CME feature. The dashed lines represent the upper limit, which was obtained from subtracting a 12-hour minimum image; and the dotted lines depict the lower limit, which was obtained from subtracting a pre-event image as the background. The average values of the upper and lower limits are denoted by the solid lines.

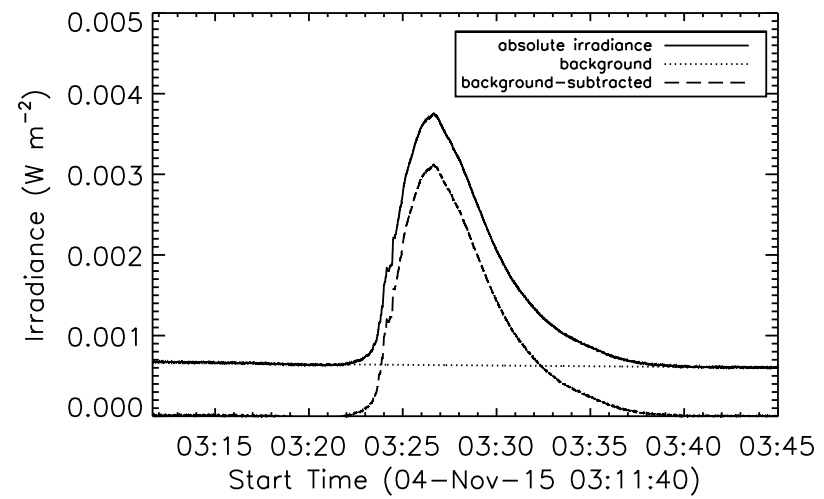

Figure 16: The irradiance in the range of 0.1-7 $\mathrm{nm}$ recorded by the SDO EVE/ESP. The solid line presents the absolute irradiance. The background and background-subtracted irradiance are delineated by dotted line and dashed line, respectively.

2012) onboard SDO. The light curves of the 0.1-7 nm waveband derived from the Extreme Ultraviolet SpectroPhotometer (ESP) is shown in Figure 16, and then used to quantify the radiative output of the EUV emission. The integration of the background-subtracted irradiance over the flare duration yields the radiated output in 0.1-7 $\mathrm{nm}$ is about $1.6 \times 10^{30} \mathrm{erg}$. The total radiated output is a few times larger than this value (Emslie et al. 2012). We can find that the flare probably consumes similar amount of magnetic energy to the CME $\left(4 \times 10^{30} \mathrm{erg}\right)$ in this event. Due to the short duration of the flare, compared with the large-scale eruptions (Emslie et al. 2012; Feng et al. 2013), the released magnetic energy of this flare is about one order of magnitude lower. Aschwanden (2016) selected $399 \mathrm{M}$ and X class flare events and obtained the parameters of the associated CMEs. We find that the CME mass and energy, in this event, lies in the lower end of their statistics. Although the absolute quantities of the flare and CME energy are smaller, the very similar partition of the flare and CME energy along with a similar multi-phase kinematics may imply that small- and largescale events share similar relationship between flares and CMEs. Although we have found some common characteristics between the small- and large-scale eruptions in terms of the flare-and-CME temporal and energetic relationships, we also need to understand what makes this event kinematically special, e.g., a very short impulsive acceleration phase, and a very high acceleration. We explore the Erupting Flux Rope model proposed by Chen (1989), in which there exists a relationship between the 
acceleration $a$ of an MFR (or a HC, its manifestation in AIA) and its geometrical size:

$$
\begin{gathered}
a=\frac{d^{2} Z}{d t^{2}} \propto\left[R \ln \left(8 R / a_{f}\right)\right]^{-2}, \\
R=\frac{Z^{2}+S_{f}^{2} / 4}{2 Z} .
\end{gathered}
$$

where $Z$ is the height of the centroid at the FR apex; $R$ is the major radius of the current channel assumed to be uniform along the MFR; $a_{f}$ is the minor radius at the footpoint which is also assumed as an invariant; $S_{f}$ is the distance of FR footpoint separation. Chen \& Krall (2003) found a scaling law related to the acceleration of CMEs and $S_{f}$ : there are two critical heights scaled with $S_{f}$ in condition that the studied structure is an MFR, and the scale is given by $Z_{*}=S_{f} / 2$ and $Z_{m} \simeq$ $1.5 S_{f}$, such that the height $Z_{\max }$ where the acceleration of the centroid of the MFR apex is maximal satisfies $Z_{*}<Z_{\max }<Z_{m}$. We speculate that $S_{f}$ is very small and eventually leads to a very low $Z_{\max }$. According to Equation (6) and (7), there is also a tendency that the smaller the major radius $\mathrm{R}$ is, the larger the acceleration of the MFR will be. Therefore, the large acceleration together with the low height at which the MFR reaches its maximal acceleration jointly produce a short duration of the impulsive acceleration phase.

We are very grateful to anonymous referee for very constructive comments and suggestions. We thank James Chen for his suggestions on the EFR model. We are also thankful to the World Data Centre of the Australian Bureau of Meteorology, Space Weather Services for the Culgoora and Learmonth radio spectrograph data. SDO is a mission of NASA's Living With a Star Program, SOHO is a mission of international cooperation between ESA and NASA. This work is supported by the NSFC grants $(11522328,11473070$, 11427803, U1731241) and by CAS Strategic Pioneer Program on Space Science, Grant No. XDA15010600, XDA15052200, XDA15320103, XDA 15320301. L.F. also acknowledges the specialized research fund from the State Key Laboratory of Space Weather for financial support. Y.S. is supported by the One Hundred Talent Program of CAS and NSFC 11473071. J.Z. is supported by US NSF AGS-1249270 and NSF AGS-1156120.

\section{REFERENCES}

\footnotetext{
Aschwanden, M. J. 2005, Physics of the Solar Corona. An Introduction with Problems and Solutions (2nd edition) -. 2016, ApJ, 831, 105

Brueckner, G. E., Howard, R. a., Koomen, M. J., et al. 1995, Solar Physics, 162, 357
}

Canfield, R. C., Hudson, H. S., \& McKenzie, D. E. 1999,

Geophysical Research Letters, 26, 627

Chamberlin, P. C., Milligan, R. O., \& Woods, T. N. 2012,

Sol. Phys., 279, 23

Chen, J. 1989, ApJ, 338, 453

Chen, J., \& Garren, D. A. 1993, Geophys. Res. Lett., 20, 2319

Chen, J., \& Krall, J. 2003, Journal of Geophysical Research (Space Physics), 108, 1410

Chen, J., \& Kunkel, V. 2010, ApJ, 717, 1105

Cheng, X., Ding, M. D., \& Fang, C. 2015, The Astrophysical Journal, 804, 1

Cheng, X., Zhang, J., Ding, M. D., Liu, Y., \& Poomvises, W. 2013, The Astrophysical Journal, 763, 43

Cheng, X., Zhang, J., Saar, S. H., \& Ding, M. D. 2012, ApJ, 761, 62

Cheng, X., Ding, M. D., Guo, Y., et al. 2014, The Astrophysical Journal, 780, 28

Dove, J. B., Gibson, S. E., Rachmeler, L. A., Tomczyk, S., \& Judge, P. 2011, The Astrophysical Journal, 731, L1

Draine, B. T., \& McKee, C. F. 1993, ARA\&A, 31, 373

Dulk, G. A., \& McLean, D. J. 1978, Solar Physics, 57, 279

Emslie, A. G., Dennis, B. R., Holman, G. D., \& Hudson, H. S. 2005, Journal of Geophysical Research (Space Physics), 110, A11103

Emslie, A. G., Dennis, B. R., Shih, A. Y., et al. 2012, ApJ, 759, 71

Fan, Y., \& Gibson, S. E. 2003, ApJL, 589, L105

Feng, L., Inhester, B., \& Gan, W. 2015a, ApJ, 805, 113

Feng, L., Wang, Y., Shen, F., et al. 2015b, ApJ, 812, 70

Feng, L., Wiegelmann, T., Su, Y., et al. 2013, ApJ, 765, 37

Gibson, S. E., \& Fan, Y. 2006, Journal of Geophysical Research (Space Physics), 111, A12103

Gibson, S. E., Fan, Y., Mandrini, C., Fisher, G., \& Demoulin, P. 2004, The Astrophysical Journal, 617, 600

Gibson, S. E., \& Low, B. C. 1998, ApJ, 493, 460

Gibson, S. E., Kucera, T. A., Rastawicki, D., et al. 2010, ApJ, 724,1133

Gopalswamy, N., Aguilar-Rodriguez, E., Yashiro, S., et al. 2005, Journal of Geophysical Research (Space Physics), 110, A12S07

Gopalswamy, N., Nitta, N., Akiyama, S., Mäkelä, P., \& Yashiro, S. 2012, ApJ, 744, 72

Guo, Y., Schmieder, B., Démoulin, P., et al. 2010, The Astrophysical Journal, 714, 343

Hong, J., Jiang, Y., Yang, J., et al. 2016, ApJ, 830, 60

Hurford, G. J., Schmahl, E. J., Schwartz, R. A., et al. 2002,

Sol. Phys., 210, 61

Illing, R. M. E., \& Hundhausen, A. J. 1983, 88, 210

Krüger, A. 1979, Geophysics and Astrophysics Monographs, 16

Kucera, T. A., Gibson, S. E., Schmit, D. J., Landi, E., \& Tripathi, D. 2012, ApJ, 757, 73

Kumari, A., Ramesh, R., Kathiravan, C., \& Gopalswamy, N. 2017, ApJ, 843, 10

Lemen, J. R., Title, A. M., Akin, D. J., et al. 2012, Sol. Phys., 275,17

Lin, R. P., Dennis, B. R., Hurford, G. J., et al. 2002, Sol. Phys., 210,3

Low, B. C., \& Hundhausen, J. R. 1995, The Astrophysical Journal, 443, 818

Lu, L., Inhester, B., Feng, L., Liu, S., \& Zhao, X. 2017, ApJ, 835, 188

Mackay, D. H., Karpen, J. T., Ballester, J. L., Schmieder, B., \& Aulanier, G. 2010, Space Science Reviews, 151, 333

Manchester, IV, W., Gombosi, T., DeZeeuw, D., \& Fan, Y. 2004, ApJ, 610, 588

Nelson, G. J., \& Melrose, D. B. 1985, Type II bursts, ed. D. J. McLean \& N. R. Labrum, 333-359

Patsourakos, S., Vourlidas, a., \& Stenborg, G. 2013, The Astrophysical Journal, 764, 125 
Prakash, O., Feng, L., Michalek, G., et al. 2017, Ap\&SS, 362, 56

Reeves, K. K., \& Golub, L. 2011, ApJL, 727, L52

Roussev, I. I., Forbes, T. G., Gombosi, T. I., et al. 2003, ApJL, $588, \mathrm{~L} 45$

Rust, D. M., \& Kumar, a. 1996, The Astrophysical Journal, 464, L199

Scherrer, P. H., Schou, J., Bush, R. I., et al. 2012, Sol. Phys., 275, 207

Schmit, D. J., Gibson, S. E., Tomczyk, S., et al. 2009, ApJL, 700, L96

Schrijver, C. J., \& De Rosa, M. L. 2003, Sol. Phys., 212, 165

Smerd, S. F., Sheridan, K. V., \& Stewart, R. T. 1974, in IAU Symposium, Vol. 57, Coronal Disturbances, ed. G. A. Newkirk, 389

Smerd, S. F., Sheridan, K. V., \& Stewart, R. T. 1975, Astrophys. Lett., 16, 23

Song, H. Q., Zhang, J., Chen, Y., \& Cheng, X. 2014, ApJL, 792, L40

Su, W., Cheng, X., Ding, M. D., et al. 2016, ApJ, 830, 70

Su, W., Cheng, X., Ding, M. D., Chen, P. F., \& Sun, J. Q. 2015, ApJ, 804, 88

Su, Y., Surges, V., van Ballegooijen, A., DeLuca, E., \& Golub, L. 2011, The Astrophysical Journal, 734, 53

Tripathi, D., Kliem, B., Mason, H. E., Young, P. R., \& Green, L. M. 2009, The Astrophysical Journal, 698, L27

Žic, T., Vršnak, B., Temmer, M., \& Jacobs, C. 2008, Sol. Phys., 253, 237
Vršnak, B. 1990, Sol. Phys., 129, 295

Vršnak, B., Aurass, H., Magdalenić, J., \& Gopalswamy, N. 2001, A\&A, 377, 321

Vršnak, B., \& Cliver, E. W. 2008, Sol. Phys., 253, 215

Vršnak, B., Magdalenić, J., Aurass, H., \& Mann, G. 2002, A\&A, 396, 673

Vršnak, B., Magdalenić, J., \& Zlobec, P. 2004, A\&A, 413, 753

Wang, H., Cao, W., Liu, C., et al. 2015, Nature Communications, 6,7008

Wang, J., Li, W., Denker, C., et al. 2000, ApJ, 530, 1071

Warmuth, A. 2015, Living Reviews in Solar Physics, 12, 3

Weber, M. A., Deluca, E. E., Golub, L., \& Sette, A. L. 2004, in IAU Symposium, Vol. 223, Multi-Wavelength Investigations of Solar Activity, ed. A. V. Stepanov, E. E. Benevolenskaya, \&

A. G. Kosovichev, 321-328

Woods, T. N., Eparvier, F. G., Hock, R., et al. 2012, Solar Physics, 275, 115

Wu, S. T., Guo, W. P., \& Dryer, M. 1997, Sol. Phys., 170, 265

Wyper, P. F., Antiochos, S. K., \& DeVore, C. R. 2017, Nature, 544,452

Zhang, J., Cheng, X., \& Ding, M.-D. 2012, Nature

Communications, 3, 747

Zhang, J., \& Dere, K. P. 2006, ApJ, 649, 1100

Zhang, J., Dere, K. P., Howard, R. A., Kundu, M. R., \& White, S. M. 2001, ApJ, 559, 452 